Niklas Lucht, Stephan Hinrichs, Larissa Großmann, Catharina Pelz, Elena Felgenhauer, Eike Clasen, Max Schwenk and Birgit Hankiewicz*

\title{
4 Synthesis of magnetic ferrogels: a tool-box approach for finely tuned magnetic- and temperature-dependent properties
}

\begin{abstract}
Multi responsive hydrogels have many potential applications in the field of medicine as well as technical fields and are of great interest in fundamental research. Here we present the synthesis and characterization of tailored magnetic hydrogels micro- as well as macrogels - which consist of iron oxide and cobalt ferrite, varying in phase and morphology, embedded in a thermoresponsive polymer. We introduce new ways to synthesize magnetic particles and revisit some common strategies when dealing with particle synthesis. Subsequently we discuss the details of the thermoresponsive matrix and how we can influence and manipulate the thermoresponsive properties, i.e. the lower critical solution temperature. Ultimately, we present the particle-hydrogel composite and show two exemplary applications for particle matrix interactions, i.e. heat transfer and reorientation of the particles in a magnetic field.
\end{abstract}

Keywords: cobalt ferrite; hyperthermia; iron oxides; magnetite; SPIONs; thermoresponsive polymer.

\subsection{Introduction}

Ferrogels combine the advantages of a gel- or polymer matrix with the magnetic properties of a ferrofluid [1-4]. As matrix materials hydrogels can be utilized to incorporate water. Many of those hydrogels can respond to external stimuli like temperature, $\mathrm{pH}$ or light. Those kinds of hydrogels are known as responsive hydrogels [4-7]. Their characteristics have a variety of potential applications in medicine and technics [2, 6-9].

An even more versatile class of materials are multiresponsive hydrogels. When the hydrogels are combined with magnetic particles, the particles can heat up the polymer matrix when exposed to an alternating current magnetic field, thus the magnetic properties of the particles influence the thermoresponsive properties of the hydrogel.

*Corresponding author: Birgit Hankiewicz, Institute of Physical Chemistry, Hamburg University, Grindelallee 117, 20146 Hamburg, Germany, E-mail: birgit.hankiewicz@chemie.uni-hamburg.de Niklas Lucht, Stephan Hinrichs, Larissa Großmann, Catharina Pelz, Elena Felgenhauer, Eike Clasen and Max Schwenk, Institute of Physical Chemistry, Hamburg University, Grindelallee 117, 20146 Hamburg, Germany

O Open Access. () 2020 Niklas Lucht et al., published by De Gruyter. (c) BY-NC-ND This work is licensed under the Creative Commons Attribution-NonCommercial-NoDerivatives 4.0 International License.

This article has previously been published in the journal Physical Sciences Reviews. Please cite as: N. Lucht, S. Hinrichs, L. Großmann, C. Pelz, E. Felgenhauer, E. Clasen, M. Schwenk and B. Hankiewicz "Synthesis of magnetic ferrogels: a tool-box approach for finely tuned magnetic- and temperature-dependent properties" Physical Sciences Reviews [Online] 2021, 6. DOI: 10.1515/psr-2019-0120 | https://doi.org/10.1515/9783110569636-004 
Those materials can be used in heat therapy for cancer treatment [10] or in controlled drug release applications [11] as well as for shape-memory actuators [3].

When an alternating magnetic field is applied, the particles can rotate either by spin-flip processes within the particles, known as Néel relaxation, or by rotation of the whole particle, known as Brownian relaxation. Which process dominates depends on the particle size and the magnetic anisotropy. The magnetic relaxation mechanism can be tuned to affect the possible heating efficiency via induction [12-14]. Hard magnetic materials like cobalt ferrite are already dominated by Brownian relaxation above $5 \mathrm{~nm}$, whereas soft magnetic particles like magnetite require larger particle sizes [14]. The shape of the particles plays an important role as well.

Anisotropic particles are more responsive to external magnetic fields compared to spherical particles due to their higher coercivity force $[15,16]$. Cubic particles are suitable particles for magnetic resonance therapy [17] or as heat mediator in general purpose $[18,19]$.

Finally, an important parameter is the magnetic moment. For most applications, a high magnetic moment is necessary. Hence, there is a demand for magnetic particles with a high saturation magnetization. Cobalt, iron and other transition-metal composites have an inherently high magnetic moment. However, they suffer from their high sensitivity to oxidation and the synthesis is often challenging [20], therefore iron oxides like magnetite and maghemite are primarily used despite their lower magnetic moment. Depending on the application, the right choice of elements used for magnetic particles is crucial, apart from their shape and size.

In the Section 4.2, we will sum up the synthesis procedures for magnetic nanoparticles with the focus on pure and mixed iron oxides in water. In addition, we will highlight the preparation of anisotropic particles with a low magnetic moment like goethite and hematite which can be oriented in very low magnetic fields due to their inherent anisotropy. In Section 4.3 we will summarize the possibilities to embed the nanoparticles within a thermoresponsive matrix. In Section 4.4 we will focus on a possible application for magnetic hydrogels: clinical hyperthermia. We will present a proof of concept for hyperthermia induced into a poly( $N$-isopropylacrylamide) (pNIPAM) matrix by cobalt ferrite nanoparticles. Section 4.5 of this report will cover the details about the experimental methods.

\subsection{Preparation of magnetic particles}

One of the main challenges during the synthesis is the stabilization right after particle formation to prevent agglomeration as early as possible. Once particles are agglomerated it is hard to separate them again. The stronger the magnetic dipole-dipole interactions are the higher the tendency to form agglomerates. Especially for biomedical as well as technological applications particles with high magnetization are necessary which must be still stable in dispersion. Particles with high magnetic 
moments like pure metals, e.g., iron and cobalt, suffer from oxidization and are sensitive to handle in general. Therefore, most common magnetic nanoparticles are the respective oxides; magnetite most prominently. Despite being an oxide magnetite is vulnerable to further oxidization into a less magnetic iron oxide phase.

Another important factor to consider is that most biomedical applications require water-based systems. There is a plethora of aqueous synthesis routes to prepare aqueous iron oxides which can be mainly divided into two categories [20]. One is the direct bottom-up synthesis of nanoparticles using iron salt precursors the other is thermal decomposition of organic iron complexes. The latter needs a very high-boiling organic solvent which makes a phase transfer as post modification step obligatory [21]. The main advantage of this method is that very narrow size distributions can be achieved and superparamagnetic iron oxide nanoparticles (SPIONs) below $20 \mathrm{~nm}$ can be produced [22]. Apart from the additional phase-transfer step required, the toxicity of the organic solvent is another drawback. The common direct aqueous approach is based on a coprecipitation method. The main advantage of this method is that the synthesis is performed in directly water and the particles can be stabilized without phase transfer [23].

Using an autoclave as the reaction vessel, the synthesis can take place at temperatures above the boiling point of water, so-called hydrothermal synthesis. Hydrothermal synthesis using an aqueous solution of simple metal salts offers a very facile, fast and low-cost procedure [24]. Additionally there is a tendency for higher crystallinity using a hydrothermal approach compared to other methods [25].

In the next section we will present the outcome of our research on how to synthesize anisotropic magnetite particles in water. We will discuss the possibility to use a reduction step to prepare magnetite and discuss cubic magnetite particles in more details. In addition, we show the potential of cobalt ferrite as a model system for Brownian relaxation.

\subsubsection{Anisotropic iron oxide particles}

A general rule of thumb for magnetic particles is that with increasing particle size the tendency to agglomerate grows. One approach to circumvent this problem is the synthesis of a low magnetic moment iron oxide species which is stabilized in solution, e.g., by synthesis of a silica-shell around the particle, to prevent agglomeration. In a second step a phase transformation to a stronger magnetic iron phase like magnetite can be performed. Hereafter we present promising precursors with the focus on anisotropic particles and delineate approaches to transform the particle phase.

\subsubsection{Goethite, hematite and akaganeite}

Literature describes many different ways to synthesize anisotropic goethite, hematite and akaganeite. Goethite is the most stable iron oxide phase. Goethite results via aging 
of iron(III)-salts at high $\mathrm{pH}$ values [26]. Depending on the temperature and concentration different shapes can be synthesized, i.e., long cylinders can be prepared keeping an iron(III)-solution for nine days at $70{ }^{\circ} \mathrm{C}$ [27]. The resulting cylinders are of about $500 \mathrm{~nm}$ in length and $10 \mathrm{~nm}$ in width (Figure 4.1A). Most of the known goethite syntheses lead to polydisperse systems (Figure 4.1B). By transferring the aging process into a hydrothermal reactor, we can gain some more control over the shape of the goethite rods $[28,29]$. Surface active substances can be used to tune the particle shape [30, 31]. In short, akaganeite (described further down) is synthesized by hydrolysis and used as a precursor to age in a hydrothermal reactor. The $\mathrm{pH}$ is adjusted to 12 and poly vinyl pyrrolidone (PVP) is added, the amount depending on the desired particle size. The hydrothermal process takes part at $160{ }^{\circ} \mathrm{C}$ under 10 bar nitrogen pressure. The reaction is stirred for $1 \mathrm{~h}$ and then heated during another hour, the temperature is kept at $160{ }^{\circ} \mathrm{C}$ for $2 \mathrm{~h}$ before cooling to ambient temperatures in an ice bath. The final product, i.e., length and width of the cylinders, depends on the stirring speed, the amount of PVP and the exact timing of PVP addition, as well as on the precursor quality. Further details will be discussed elsewhere [32].

Hematite can be prepared at weakly alkaline conditions [26]. The typical crystalline structure of hematite is cubical, therefore it is the easiest to prepare cubes [33]. To demonstrate this, an iron-(III)-chloride solution has been aged for eight days. Depending

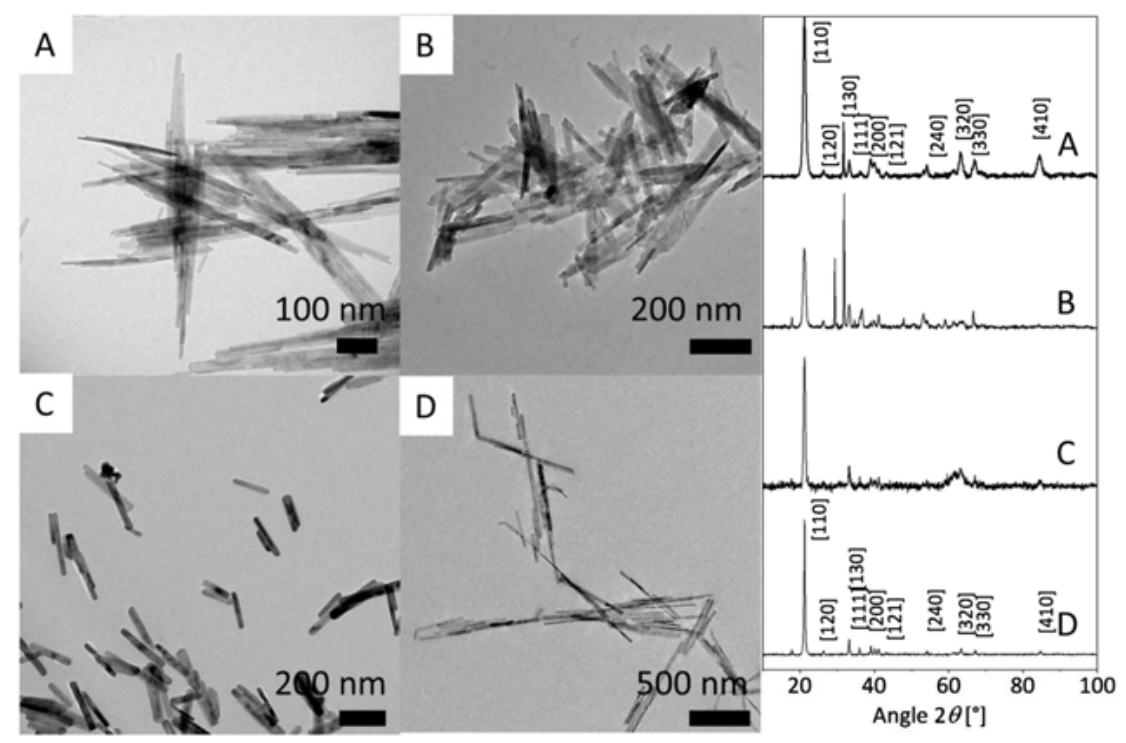

Figure 4.1: TE micrographs (left) and XRD (right) of anisotropic goethite nanoparticles, (A) aged at high $\mathrm{pH}$ at $70^{\circ} \mathrm{C}$ for nine days, (B) aged at high $\mathrm{pH}$ over several days at room temperature, (C) aged in a hydrothermal reactor at $\mathrm{pH} 12$ over $4 \mathrm{~h}$ at $160^{\circ} \mathrm{C}(\mathrm{D})$ aged in a hydrothermal reactor at $\mathrm{pH} 12$ over $4 \mathrm{~h}$ at $160{ }^{\circ} \mathrm{C}$ with PVP. The peaks of the XRD pattern can be indexed by the goethite reference indicated in black. 
on the temperature and the initial aging solution different particle sizes can be prepared [34]. This yields cubic particles between $30 \mathrm{~nm}$ and $1 \mu \mathrm{m}$ (Figure 4.2A). XRD confirms phase pure hematite. Compared to goethite, monodisperse particles are easier to prepare.

The most common approach for the synthesis of anisotropic hematite is to use phosphate ions. Phosphate ions have a strong coordination to the c-axis of the hexagonal crystal system, therefore the particle shape results in spindles (Figure 4.2B) [33]. The aspect ratio of hematite spindles can be varied via the phosphate ion concentration $[35,36]$.

Surface active substances, typically cetyltrimethylammonium bromide (CTAB) or polyethylene glycol (PEG), play a huge role in particle shape during their formation [37, 38]. As an example a $0.2 \mathrm{M}$ iron(III)-solution with $0.02 \mathrm{M} \mathrm{CTAB}$ at $120{ }^{\circ} \mathrm{C}$ creates cylinders with a length of about $480 \mathrm{~nm}$ and width of about $60-80 \mathrm{~nm}$ (Figure 4.2C). Analogous the addition of PEG also yields cylindrical structures. The aspect ratio of the particles is directly influenced by the molecular weight of the PEG. In a hydrothermal reactor, cylindrical particles between $500 \mathrm{~nm}$ and $1 \mu \mathrm{m}$ length and a width of about $20 \mathrm{~nm}$ can be synthesized within $24 \mathrm{~h}$ at $150{ }^{\circ} \mathrm{C}$ (Figure 4.3A). Here a mixture of a magnetite phase and a hematite phase is produced (Figure 4.3A).
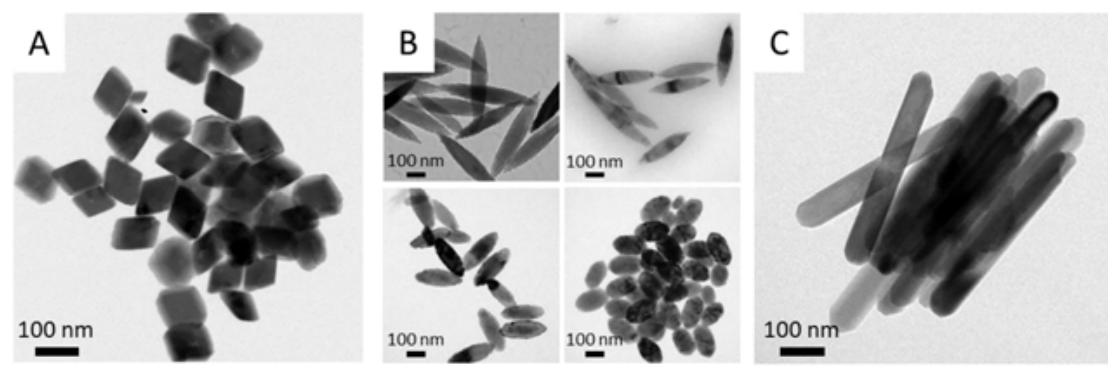

Figure 4.2: TE micrographs of hematite particles, (A) aged at lower $\mathrm{pH}$ for eight days at $100^{\circ} \mathrm{C}$, (B) prepared with different amounts of hydrogen phosphate at $100{ }^{\circ} \mathrm{C}(\mathrm{C})$ with $\mathrm{CTAB}$ at $120^{\circ} \mathrm{C}$.
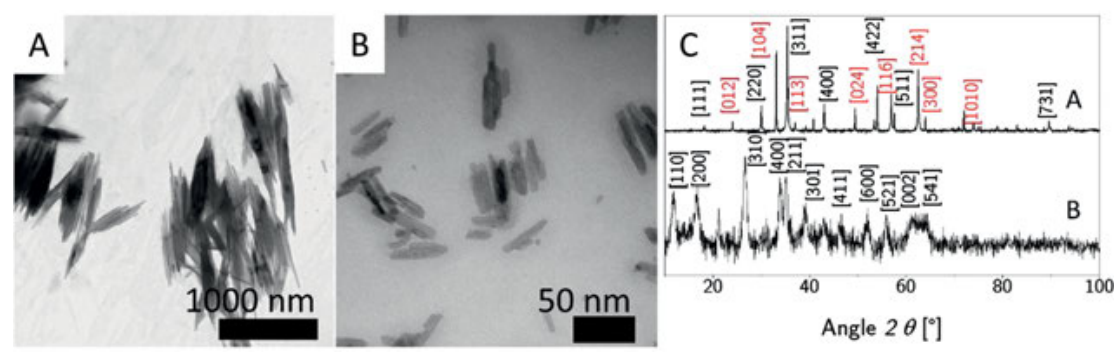

Figure 4.3: TE micrographs of $(A)$ magnetite with hematite impurities in the presence of $P E G$ and (B) $\beta$ - $\mathrm{FeOOH}$ and (C) XRD for magnetite with hematite (A) and $\beta$ - $\mathrm{FeOOH}(B)$ impurities. The peaks are indexed in black for magnetite and in red for hematite according the references. 
Akaganeite is mostly an intermediate phase at low temperatures during the synthesis to goethite or hematite (Figure 4.3B), it consists of cylinders with a length of about 20-50 $\mathrm{nm}$ and width of about $5 \mathrm{~nm}$ [39].

Further synthetic approaches for magnetic anisotropic particles like cubes [34], wires [40], propellers [41] and plates are known from literature [42, 43]. However to the best of our knowledge not many hydrogels are known with embedded anisotropic particles [44, 45].

\subsubsection{Reduction to magnetite-keeping the shape?}

To increase the magnetic moment, iron(III)-oxides can be reduced to magnetite which has a higher magnetic moment compared to goethite and hematite. Magnetite can be oxidized to maghemite which is a more stable iron oxide phase, with a weak oxidizing agent in a second step. This has already been done for hematite spindles [46, 47]. However, the reduction of hematite takes place at high temperatures $\left(400{ }^{\circ} \mathrm{C}\right)$ under hydrogen atmosphere. The oxidization can then take place using nitric acid [48].

The disadvantage is that the particles need to be redispersed in water after the reduction. To achieve this, the particles are typically covered by a silica shell $[49,50]$. Another possibility is the transfer of the process to hydrothermal conditions [51]. Crassou et al. [52] reported a method to stabilize the particles with a thin silica layer and to subsequently incorporate them into a hydrogel. The thickness of the shell can be tuned via the amount of tetraethoxysilane (TEOS) (Figure 4.4A-C). This procedure can be adapted to goethite and akaganeite by following Graf et al. [49]. As a reduction agent sodium borohydride, hydrazine and sodium phosphite were already reportedly used for aqueous systems [40, 53-57]. The redox potential (see Table 4.1) shows that iron ions
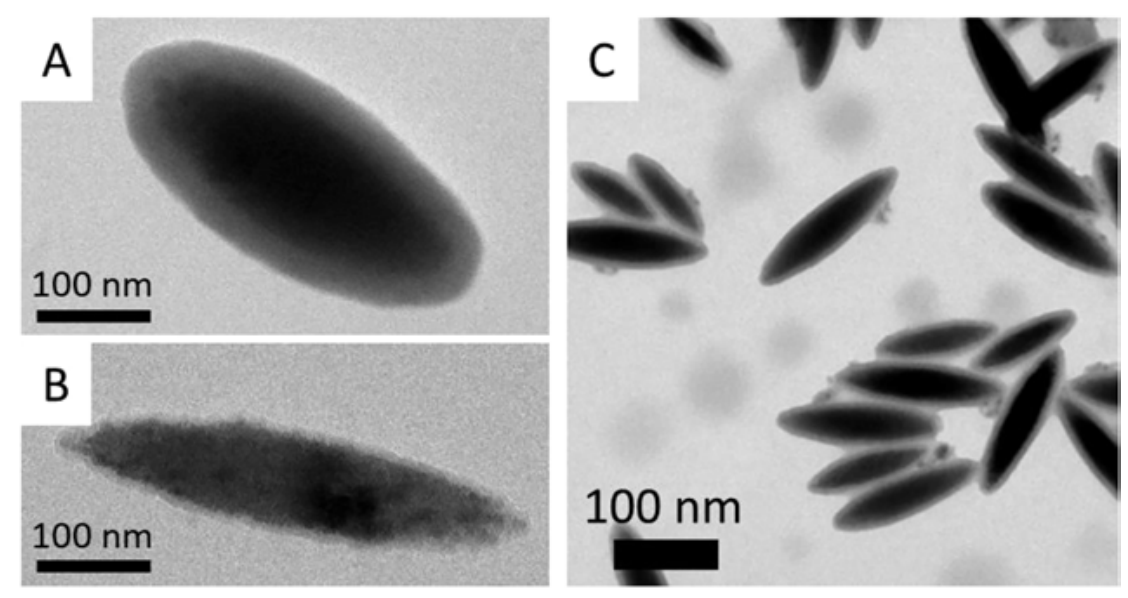

Figure 4.4: TE micrographs of the silica covered hematite spindles with different thicknesses of the silica shell from about 5 to $40 \mathrm{~nm}(\mathrm{~A}-\mathrm{C})$. 
Table 4.1: Standard redox potential of the reducing agents and of iron.

\begin{tabular}{llr}
\hline Substance & Reaction & Standard potential E0/V vs. NHE 25 ${ }^{\circ} \mathrm{C}$ \\
\hline $\mathrm{Fe}^{3+}$ & $\mathrm{Fe}^{3+}+\mathrm{e}^{-} \rightarrow \mathrm{Fe}^{2+}$ & 0.77 [58] \\
$\mathrm{Fe}^{2+}$ & $\mathrm{Fe}^{2+}+2 \mathrm{e}^{-} \rightarrow \mathrm{Fe}$ & $-0.44[57]$ \\
$\mathrm{NaBH}_{4}$ & $\mathrm{BH}_{4}^{-}+4 \mathrm{OH}^{-} \rightarrow \mathrm{BO}_{2}^{-}+2 \mathrm{H}_{2} \mathrm{O}+2 \mathrm{H}_{2}+4 \mathrm{e}^{-}$ & $-1.73[57]$ \\
$\mathrm{N}_{2} \mathrm{H}_{4}$ & $\mathrm{~N}_{2} \mathrm{H}_{4}+4 \mathrm{OH}^{-} \rightarrow \mathrm{N}_{2}+4 \mathrm{H}_{2} \mathrm{O}+4 \mathrm{e}^{-}$ & $-1.16[57]$ \\
\hline
\end{tabular}

can even be reduced to their elementary state. Nevertheless oxygen from the air often results in a reoxidation which needs to be taken into account for the synthesis conditions [57]. Therefore all reductions need to be performed under nitrogen atmosphere.

With sodium borohydride the particles could be reduced to magnetite, however the resulting shape strongly depends on the rate of sodium borohydride addition.

A fast addition rate $(40 \mathrm{~mL} / \mathrm{h}$ ) leads to spherical particles (Figure $4.5 \mathrm{~A}$ ) and a slow addition rate $(5 \mathrm{~mL} / \mathrm{h})$ can partially keep the particle shape (Figure $4.5 \mathrm{~B}$ ). A slow addition rate does not lead to complete reduction of the product although the same total amount of sodium borohydride is used. Not only the addition rate but also the $\mathrm{pH}$, the ratio of $\mathrm{Fe}^{3+}$ to the reduction agent, pressure and temperature take an important role in the reductive synthesis of magnetite.

Although it proved to be difficult to keep the shape in a reproducible way, there are several synthetics possibilities to use the anisotropic particles from Section 4.2.1.1 made of goethite, hematite or akaganeite to produce magnetite particles via reduction with anisotropic shape. Exemplarily we will show it on cubic particles in the following section.
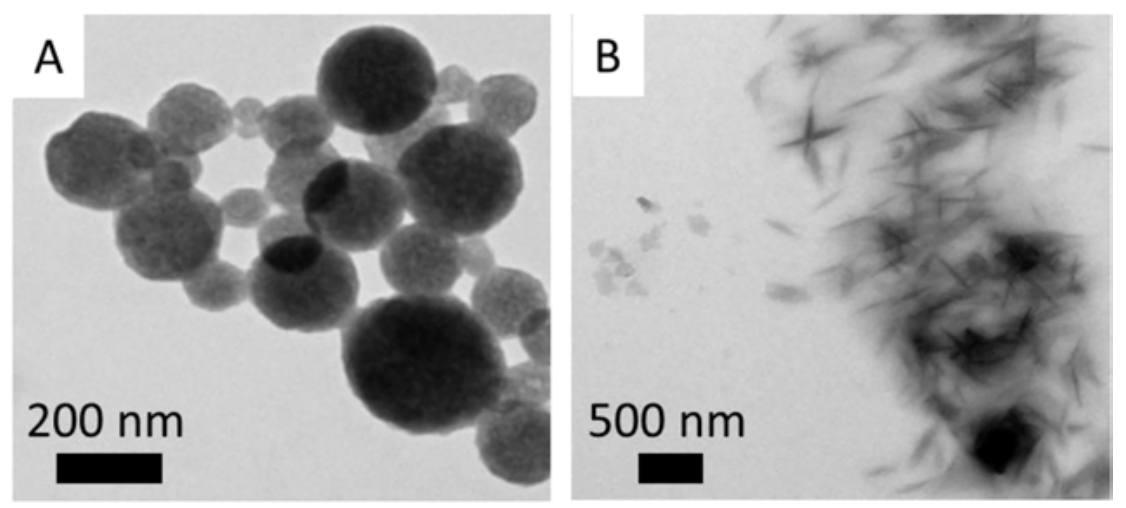

Figure 4.5: TE micrographs after reduction of iron(III)-oxide with sodium borohydride with fast (A) and slow addition rate (B) of the reduction agent. 


\subsubsection{Cubic magnetite}

One reduction approach was deemed especially promising to produce cubic magnetite particles [59]. In the first step a precursor consisting of akaganeite is synthesized by treating an iron(III)-solution with $\mathrm{NaH}_{2} \mathrm{PO}_{4}$ at $80{ }^{\circ} \mathrm{C}$ [34]. The resulting precursor particles consist of small cylinders (20-55 nm length) (similar to Figure 4.3B). In the second step of the synthesis the precursor is reduced to magnetite by hydrazine. In order to optimize this reaction, we tested a whole array of parameters, employed in various research articles [38, 60-62]. We worked at temperatures between 80 and $160^{\circ} \mathrm{C}$, varied the ratio of iron educt to reducing agent, the concentration of the akaganeite precursor, the variety and concentration of surfactants as well as the duration of the whole reaction. We observed the best results when using a $0.2 \mathrm{M}$ precursor solution with hydrazine in a ratio of 12:1 iron:hydrazine. The reaction was heated to $160{ }^{\circ} \mathrm{C}$ and held for $24 \mathrm{~h}$ while stirring. As structure forming agent the leftover $\mathrm{NaH}_{2} \mathrm{PO}_{4}$ from the precursor particles is used. This reaction produced weakly anisotropic, but phase pure magnetite cubes with an edge length of about $25 \mathrm{~nm}$ (Figure 4.6A and B). We studied the synthesis during different time frames and observed that during the reduction after 5-10 $\mathrm{h}$ big agglomerates form in the reaction medium (Figure 4.7A and $\mathrm{B}$ ). These dissolve over the course of the reaction to form spherical particles after around $20 \mathrm{~h}$ (Figure 4.7C).

Afterward these particles convert to a cubic shape consisting of pure magnetite (Figure 4.7D-F). X-ray diffraction revealed that during the early stages of the reaction a goethite rich phase is formed which is subsequently reduced to magnetite (Figure 4.6B).

This reaction was scaled from a $25 \mathrm{~mL}$ reactor up to a $200 \mathrm{~mL}$ reactor and even to $500 \mathrm{~mL}$. The up-scaled process yields magnetite particles of the same dimensions and with the same phase purity as the $25 \mathrm{~mL}$ version. We even observed a decrease in polydispersity, which is caused by the increased pressure during the reaction, caused by the increased amount of hydrazine paired with the rapid dissolution of $\mathrm{NaOH}$. The product contained cubic/rhombic magnetite in the dimensions of $25 \mathrm{~nm}$ edge length (Figure 4.6A) with a saturation magnetization of about $70 \mathrm{Am}^{2} / \mathrm{kg}$ (Figure 4.6C) which
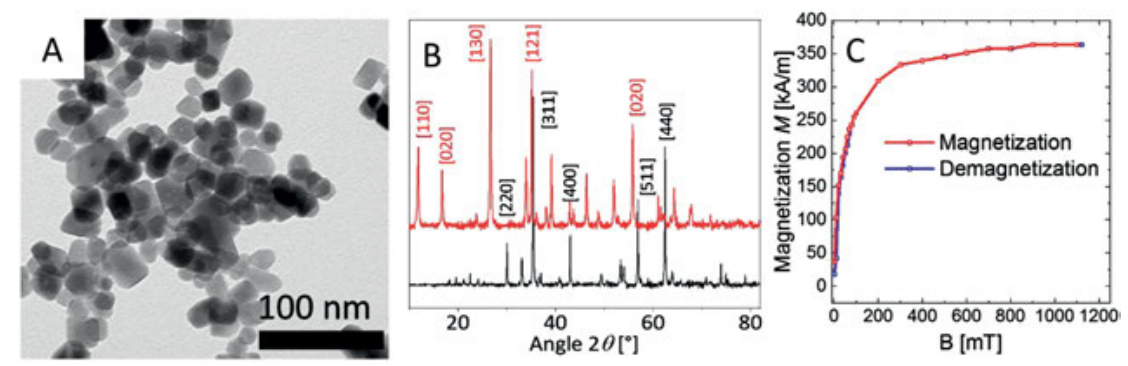

Figure 4.6: Cubic magnetite after reduction from an akaganeite precursor (A) TE micrograph (B) and XRD before (red) and after the reduction (black), peaks are indexed accordingly with akaganeite in red and magnetite in black, as well as static magnetization curve (C) of the particles in A. 


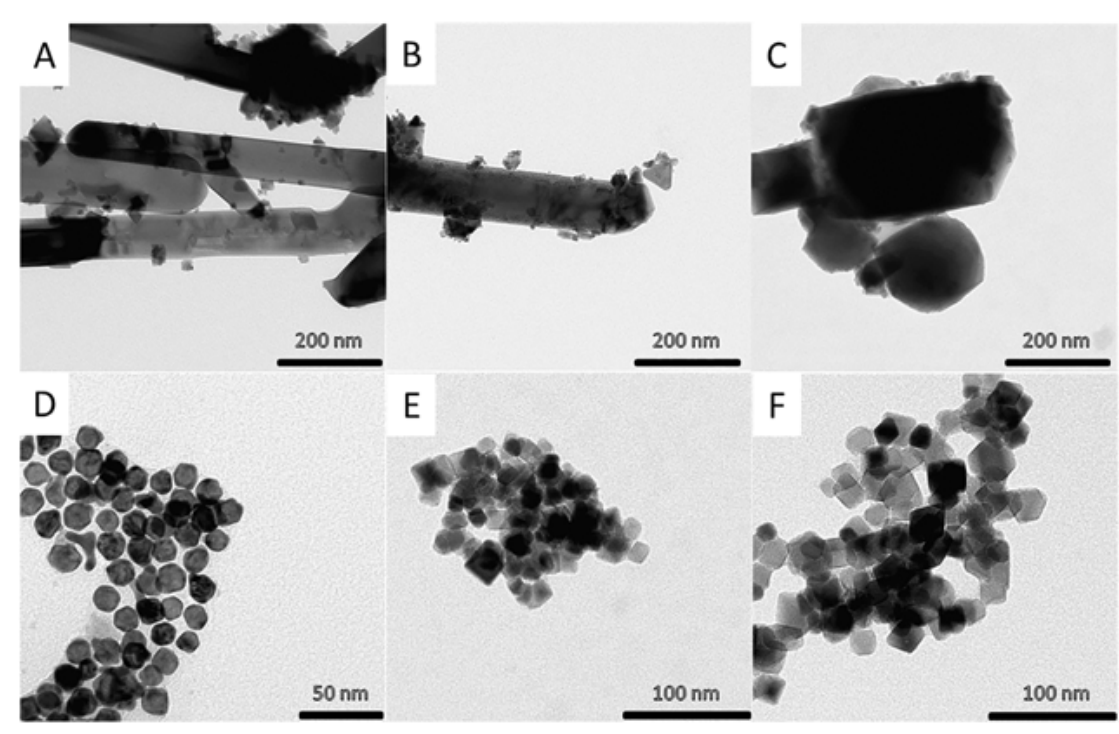

Figure 4.7: Reaction time-dependent TE micrographs study of the akaganeite reduction with $5 \mathrm{~h}(\mathrm{~A})$, $10 \mathrm{~h}(\mathrm{~B}), 15 \mathrm{~h}(\mathrm{C}), 20 \mathrm{~h}(\mathrm{D}), 48 \mathrm{~h}(\mathrm{E})$ and $72 \mathrm{~h}(\mathrm{~F})$ reaction time. All measurements were taken from single, isolated reactions.

is about $75 \%$ of the bulk magnetization. This up-scaled synthesis allowed for approx. $0.5 \mathrm{~g}$ of magnetite per reaction.

\subsubsection{Multicore particles}

Our hydrothermal treatment allows for an easy and universal conversion of akaganeite to magnetite by reduction and in some cases prior oxidation of an iron salt. We succeeded to conduct a quantitative conversion from akaganeite to magnetite without the creation of a harmful by-product. Further experiments suggest that this reaction pathway is indeed universally applicable to convert any akaganeite impurities to magnetite.

The material of interest in this case are multicore particles (Figure 4.8C) [63, 64]. Those multicore particles are precipitated with a slow addition of sodium hydrogen carbonate (about $450 \mu \mathrm{l} / \mathrm{min}$ ). However, using such slow addition rate leads to some impurities of various iron oxide phases. Therefore, we treated the sample by adding hydrazine in a 12:1 ratio iron:hydrazine and put it into a Teflon lined stainless steel hydrothermal reactor. The reactor was heated to $160{ }^{\circ} \mathrm{C}$ over the course of $2 \mathrm{~h}$ while stirring at $500 \mathrm{rpm}$.

These conditions were kept for $24 \mathrm{~h}$ before the reactor was cooled down to room temperature and the black precipitate was transferred into a beaker. The precipitate was highly magnetic and was washed three times by magnetic decantation until the $\mathrm{pH}$ of the supernatant was neutral. 

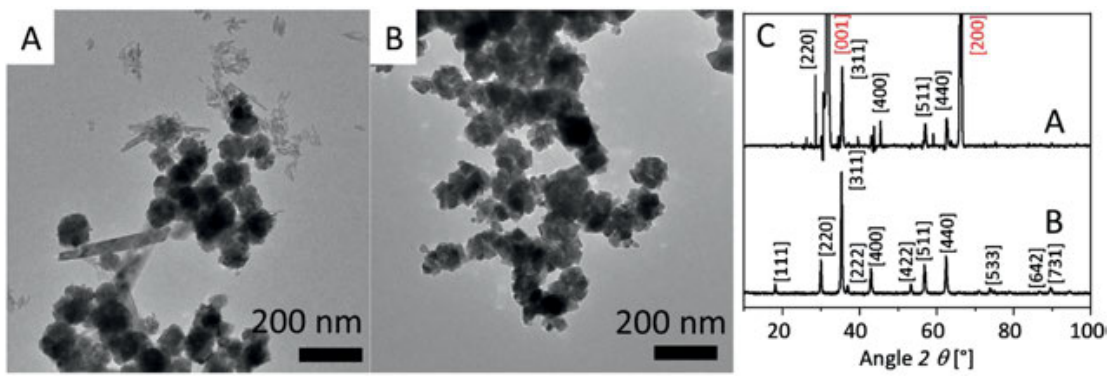

Figure 4.8: Multicore particles before hydrothermal treatment (A) and after (B). TE micrograph with different phases visible prior to hydrothermal treatment. After the treatment only one species is present (C). (C) XRD of the multicore particles before (A) and after (B) the hydrothermal treatment. The peaks are indexed in black for magnetite and in red for akaganeite impurities. They further show the phase purity after hydrothermal treatment.

The product was characterized by TEM and XRD directly after the synthesis (Figure 4.8). The resulting multicore particles consist of cubic and spherical particles alike, all of them pure magnetite as indicated by XRD (Figure 4.8C). This result indicates that this method is capable of transforming iron oxide phases to magnetite without disrupting the multicore cohesion. This can be used to improve the magnetic properties of a wide variety of mixed phase iron oxide samples that would be otherwise unusable.

\subsubsection{Cobalt ferrite particles}

Cobalt ferrite is a ferrimagnetic mixed ferrite consisting of a stoichiometric composition of one cobalt and two iron ions. While cobalt ferrite has the same crystalline structure as magnetite and is ferrimagnetic as well the size difference between the cobalt and the iron atoms results in a very high anisotropy energy resulting in cobalt ferrite being hard-magnetic with a very high coercive force in bulk. In nanomaterials this manifests in a dominating relaxation mechanism in dependence of particle size. While magnetite particles can grow well above $20 \mathrm{~nm}$ before being Brownian relaxation dominated, cobalt ferrite can already be Brownian dominated at particle sizes around $10 \mathrm{~nm}$ [14]. This makes cobalt ferrite a very interesting system to compare relaxation mechanisms of particles and a good material for hyperthermia (as discussed in Section 4.4). Cobalt ferrite also has a synthetic advantage, due to being a mixed ferrite it is far more unlikely to have phase impurities. When synthesizing magnetite, a typical problem is the partial oxidation to maghemite as an impurity during the precipitation process. Another possible, unwanted side effect is the full oxidation of magnetite to the less magnetic hematite when storing the particles.

Our cobalt ferrite is synthesized in a straightforward coprecipitation method derived from Nappini et al. [65]. $7.6 \mathrm{~g}$ cobalt(II)-nitrate and $17.3 \mathrm{~g}$ iron(III)-chloride are 
dissolved in $100 \mathrm{~mL}$ Millipore water. Two milliliter concentrated nitric acid is added and the solution is heated to the boiling point. Four-hundred milliliter of $1 \mathrm{M} \mathrm{NaOH}$ solution are prepared and heated to the boiling point. The boiling salt solution is then rapidly transferred into the $\mathrm{NaOH}$ solution under vigorous stirring. The temperature is held for another $90 \mathrm{~min}$ before cooling down the solution to room temperature. The particles are separated by magnetic decantation, washed twice with water and finally suspended in $40 \mathrm{~mL} 2 \mathrm{M} \mathrm{HNO}_{3}$. The suspension is then heated to the boiling point and a boiling mixture of $56 \mathrm{~mL}$ iron(III)-chloride and $28 \mathrm{~mL}$ cobalt(II)-nitrate is added rapidly under vigorous stirring. After $30 \mathrm{~min}$, the solution is repeatedly washed with water through magnetic decantation until a neutral $\mathrm{pH}$ of the supernatant is reached. The particles are then dispersed in $25 \mathrm{~mL} 0.25 \mathrm{M}$ aqueous tetramethylammonium hydroxide solution and slightly stirred overnight. The mass percentage of the product is determined through gravimetry. For the final stabilization step, the particle solution is added to a $100 \mathrm{mM}$ solution of citric acid with a target weight percentage of about $0.25 \mathrm{wt} \%$ and are stirred for at least $2 \mathrm{~h}$. After another magnetic separation step, the particles are dispersed in the same volume of $20 \mathrm{mM}$ trisodium citrate solution and are stirred for another hour. Lastly, the volume was reduced by about $75 \%$ through evaporation and the concentrated solution dialyzed against Millipore water for one week. The weight percentage of the final product is once again determined through gravimetry.

The cobalt ferrite particles mainly used in this project have a varying median size between 15 and $20 \mathrm{~nm}$, the exemplified TE micrograph in Figure 4.9A shows particles with $18 \pm 4 \mathrm{~nm}$. The particles are quite polydisperse and have a rough surface which is fabricated during the synthesis to increase stability in solution. The particles are stable for months in water.
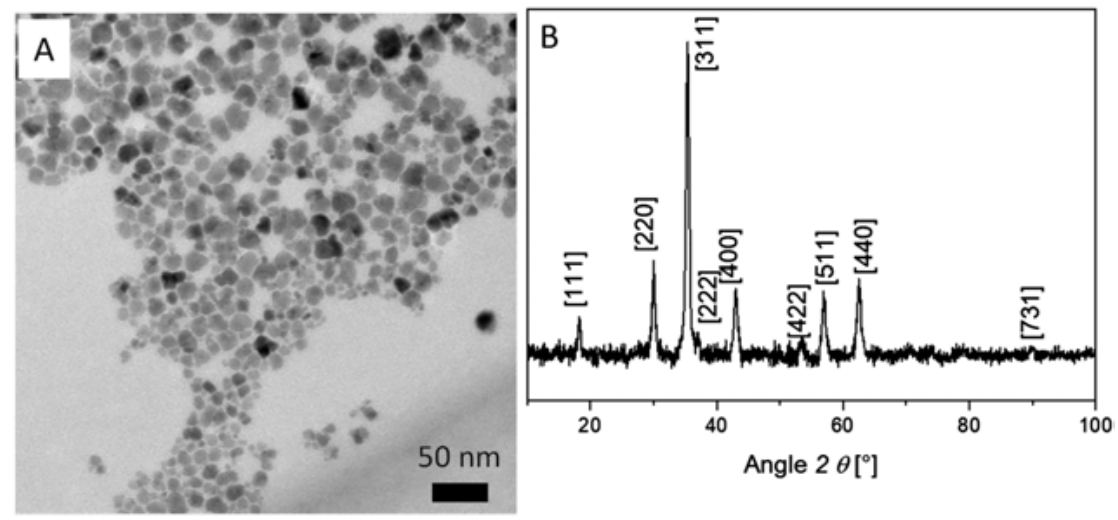

Figure 4.9: (A) TE micrograph and (B) XRD of the prepared phase-pure cobalt ferrite particles. The black indices were set by a cobalt ferrite reference. 
The nanoparticles are phase pure as shown in Figure 4.9B. Utilizing the Scherrerequation a crystallite size $15 \pm 3 \mathrm{~nm}$ is derived which is a bit smaller compared to the TEM result due to the surface roughening which leads to amorphous domains at the outer layer of the particles. The particles have high saturation magnetization of about $52 \mathrm{Am}^{2} / \mathrm{kg}$ [66]. The saturation moment of cobalt ferrite is very comparable to magnetite of the same particle size. This fact makes it very interesting to compare particle species as magnetite below $20 \mathrm{~nm}$ is typically still dominated by Néel relaxation while cobalt ferrite is already dominated by Brownian relaxation which has been shown by Draack et al. [67]. The comparatively small particle size needed for Brownian dominated relaxation is also very interesting for the application in hyperthermia. A detailed study on the dynamics of cobalt ferrite in viscoelastic media are done within the priority program SPP 1681 from the groups of Tschoepe et al. [68]. Similar mobility investigation are done for magnetite in a dextran matrix-as biocompatible composites-within the priority program [69]. There are several publications raising qualms about the biocompatibility of nanoparticles in general as it was shown that nanoparticles can easily penetrate most bodily membranes given their small size [70]. Cobalt ferrite especially raises suspicion due to its cobalt content. Elemental cobalt is a very toxic material. Cobalt ions can exchange calcium ions in the body blocking intracellular $\mathrm{Ca}^{2+}$ binding proteins, moreover, cobalt can also induce hypoxia. To alleviate those suspicions initial toxicological assessments of our particle system were conducted with Jurkat cells. In the first $24 \mathrm{~h}$ there was no apparent cytotoxicity up to concentrations of $400 \mu \mathrm{g} / \mathrm{mL}$. After $48 \mathrm{~h}$ cobalt ferrite expresses significant toxicity above $100 \mu \mathrm{g} / \mathrm{mL}$ which can be reduced by adding a silica shell to the particles [66].

\subsection{Magnetic hydrogels}

One application of multi responsive hydrogels is a controlled drug release for medical purposes. Carbon, magnetic nanoparticles or gold nanoparticles are often used to manipulate the hydrogel matrix as switches for the coil-to-globule transition. The triggers for the aforementioned switch would be radio frequency, an alternating magnetic field or light. Recently Satarkar et al. [71] highlighted the developments in those remote controlled biomaterials.

In general hydrogel systems can be distinguished by their size into micro- and macrogels (Figure 4.10). Microgels with a single particle as core are often called coreshell-microgels (Figure 4.10A) [59]. Another variant are systems with a distribution of nanoparticles over the whole microgel particle (Figure 4.10B) [72]. Macrogels can be either cross-linked microgel particles or bulk-hydrogels [71]. The synthesis process for the macrogel decides if the particles are mobile $[69,73,74]$ or fixated in the network [71, 74-76]. A comparative study of both macrogels was done by Messing et al. [74] of cobalt ferrite within ferrohydrogels. 
A

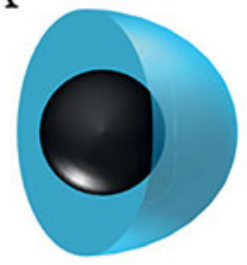

B

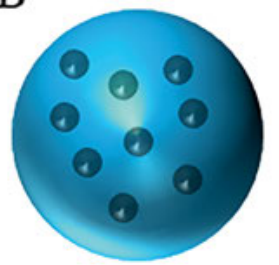

$\mathrm{C}$

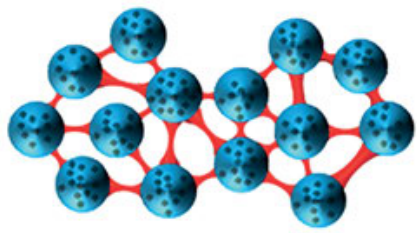

Figure 4.10: (A) Nanoparticles (black) covered with a hydrogel (blue). (B) Nanoparticles embedded in microgel microparticles. (C) Macrogel with embedded nanoparticles, red depicts a crosslinker. The scale of the particles increases from left to right. Left shows a few nanometers in total size and $\mathrm{C}$ up to the millimeter range.

We will discuss magnetic core-shell microgels (Figures 4.10A and 4.11A) and macrogels which are cross-linked microgel particles (Figure 4.11B and C). The microgel particles are able to diffuse and to rotate freely. In case of the macrogels we distinguish two types of behavior: fixed nanoparticles on network knots and free particles in the network. In this former case the core-shell microgels (Figure 4.11A) will be cross-linked additionally to a macrogel (Figure 4.11B).

In this case the particles are hindered in rotation as well as in diffusion. The embedded nanoparticles are typically covered with an additional thin silica layer. Another type of macrogel network does not completely hinder the particles in their mobility (Figure 4.11C). In this case a thermoresponsive cross-linked microgel is synthesized independently of nanoparticles which are added in a second, separate step. While the diffusion of the particles is hindered by the gel network, the particles-if small enough-can still freely rotate [73].

pNIPAM is a common and widely researched thermoresponsive polymer that has the inherent advantage that the lower critical solution temperature (LCST) lies close the human body temperature with $34^{\circ}$. The LCST describes a temperature above which two

A
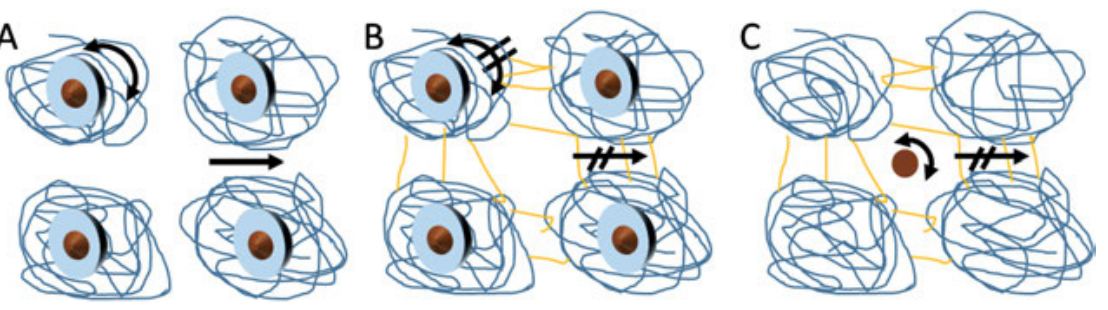

Figure 4.11: Silica coated nanoparticles (brown in light blue spheres) with random coils of a thermoresponsive polymer. The microgel particles can freely rotate and translate inside the solvent (A). When the particles are cross-linked into a macrogel (yellow lines depict the crosslinker) the translation and rotation are hindered (B). A microgel and particles can be synthesized separately and mixed prior to the crosslinking step leading to immobile particles that can still rotate (C). 


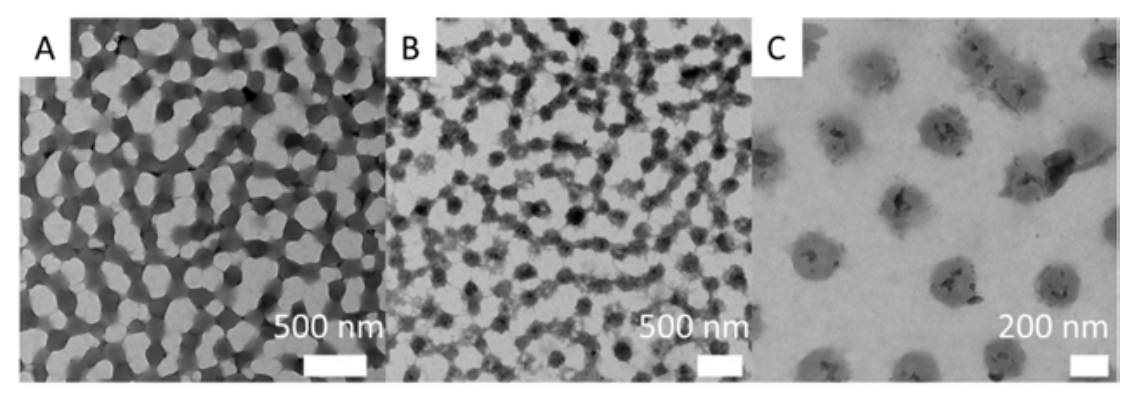

Figure 4.12: TE micrographs of macrogel particles with varying crosslinker densities, (A) $0.1 \%$, (B) $0.05 \%$, (C) $0.025 \%$ total mass of crosslinker.

substances (gel and solvent) are no longer miscible and a polymer undergoes a coil-toglobule transition, to minimize its surface area. Usually an additional crosslinking agent is used to improve the reversibility of the coil-to-globule transition. The most common crosslinking agent for acrylamides is $N, N^{\prime}$-methylene-bis-acrylamide (BIS). The amount as well as the distribution of the crosslinker within the microgel affects the swelling behavior. Varga et al. [77], showed that a homogenous distribution leads to a larger swelling ratio than a heterogeneous distribution at low temperature. The influence of the crosslinker on the LCST is quite low.

\subsubsection{Tuning of the LCST}

The LCST of pNIPAM cannot be tuned in a wide range. As an example of a more versatile matrix material copolymers like poly[ $N$-acryloyl piperidine-random- $N$-acryloylpyrrolidine] allow the tuning of the LCST in a wide range between $3^{\circ} \mathrm{C}$ and $45^{\circ} \mathrm{C}$ by varying the ratio of the monomers before the polymerization [78]. Another promising material class is poly[oligo(ethylene oxide)] which is biocompatible. The LCST of these polymers can be manipulated by adjusting the side chain length of the oligo ethylene oxide [79].

The right choice of material and a good synthesis control have the potential to create highly functional and tight fit materials. For medical applications an LCST in the range between 37 and $40^{\circ} \mathrm{C}$ would be ideal, for other application the ideal LCST could be different.

\subsubsection{Core-shell hydrogel as matrix}

A possible route to synthesize core-shell microgels is described by Karg [80, 81]. In a first approach silica nanoparticles are used as cores. The silica core is functionalized with 3-(trimethoxysilyl)propyl methacrylate (TPM) and then covered with a pNIPAM 
shell. The general swelling behavior and the LCST do not change significantly when altering the crosslinker density. This has the neat side effect that the hydrodynamic volume of the particles can be tuned independently of the thermoresponsiveness by varying the crosslinking density, i.e., the ratio of BIS and NIPAM as well as the distribution of the BIS inside the gel which has also direct influence on the temperaturedependent gel size [82].

\subsubsection{Macrogel matrix}

\subsubsection{Core-shell macrogels}

Starting from a core-shell microgel, the transition to a macrogel is rather easy. After the particles were covered by silica and while pNIPAM was polymerized onto the TPM functionalization, the NIPAM molecules were densely crosslinked with BIS to form a tight microgel sphere. The outside of this sphere was then functionalized by allylamine (AA) to provide amine functional groups which are necessary for the crosslinking into a macrogel. These amine functions are then crosslinked by glutaraldehyde (GA) at temperatures above the LCST to form a viscous macrogel [83].

\subsubsection{Macrogels}

Another approach toward particle loaded macrogels was taken by Nack et al. [73], here the microgel is made of pure pNIPAM crosslinked directly by glutaraldehyde. This synthesis is done above the LCST, so pNIPAM particles are in their shrunken state and form dense spheres that are subsequently crosslinked by GA. GA forms a bond between two NIPAM Amino groups but this bond exists in a rather unstable equilibrium, which means that the crosslinks can be easily rearranged [84]. The degree of crosslinking, i.e., the density of the polymer network, can be regulated by the GA content (Figure 4.12). Since this reaction is also done above the LCST, sodium dodecyl sulfate (SDS) is deployed to keep the polymerizing NIPAM chains in solution [85]. The size of the forming polymer globuli during the synthesis is determined by the concentration of SDS given. We used this to tune the size of the microgel spheres inside the macrogel (Figure 4.13).

\subsubsection{Magnetic particles fixed within the network-magnetic core-shell macrogels}

To synthesize core-shell microgels with magnetic nanoparticles as cores, the nanoparticles are first covered by a silica shell $[49,50]$. Then the particles are treated as described above. A first demonstration of magnetic particles fixed in a thermoresponsive 

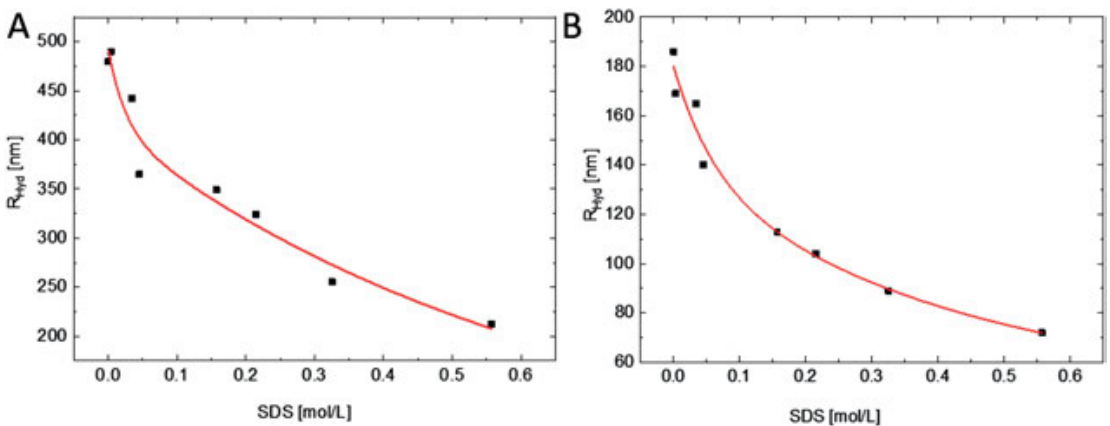

Figure 4.13: Hydrodynamic radii of pNIPAM spheres in dependence of the SDS concentration during synthesis above (A) and below (B) the LCST. The red lines are a guide to the eye roughly extrapolated as an exponential decay.

polymer was done with hematite cubes [59]. First the hematite cubes are synthesized like described by Ozaki [86] (Figure 4.2A in Section 4.2.1.1) and then covered by a thin silica layer functionalized with TPM. To grow the pNIPAM chains, a radical polymerization was performed. In addition to NIPAM the crosslinker BIS was used and the surface was covered with AA [80, 81]. As described above, the amino groups of the AA are used to crosslink the microgel to a macrogel using glutaraldehyde. This macrogel still has the same thermoresponsive behavior as the microgel. At low temperature, the microgel is swollen and has a radius of about $172 \mathrm{~nm}$. Above the LCST at about $33^{\circ} \mathrm{C}$, the microgel particles collapse and the size shrinks to $55 \mathrm{~nm}$.

This transition is also visible with the naked eye. Above the LCST, the microgel turns turbid or milky (Figure 4.14A). This is also directly visible in the macrogel (Figure 4.14B). The gelation process can be investigated by rheology in-situ [59]. The higher the temperature the faster the gelation process works. The same procedure is also possible for other magnetic cores [59].

\subsubsection{Magnetic particles mobile within the network}

An additional property of form-anisotropic particles is that they align in a magnetic field, i.e., the hematite spindles orient perpendicular to their length axis within the field

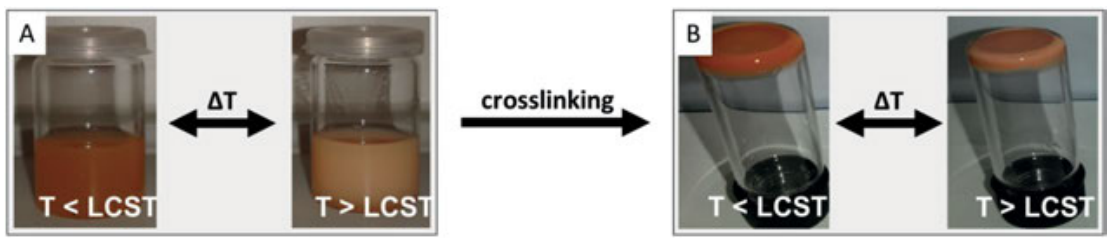

Figure 4.14: Photograph of liquid microgel particles below and above the LCST (A) and after crosslinking to a solid macrogel (B). 
$[35,36,87]$. If they were put into a macrogel, the hematite particles are hindered in their orientational behavior [73]. Investigation on the mobility of hematite spindles were done from groups of Wende and Schmidt $[76,88]$ within the priority program 1681 . This can be similarly achieved using goethite cylinders which should orient parallel in very low magnetic fields $(<35 \mathrm{mT})$ and perpendicular at higher field strengths [43, 89]. Goethite particles were synthesized according to Section 4.2.1.1 (Figure 4.1C). According to TEM, the particle length was about $210 \pm 61 \mathrm{~nm}$ and the particle width was about $27 \pm 9 \mathrm{~nm}$, resulting in an aspect ratio of about 8 . At a field strength of about $550 \mathrm{mT}$, the goethite particles align, which is visible as an anisotropic pattern in the small angle X-ray scattering data (Figure 4.15A). Compared to an isotropic pattern (indicated by a circle in the data) the minimum of the intensity shifts to higher $q$-values perpendicular to the field and to lower $q$-values parallel to the field. By averaging over a defined wave vector range, i.e., between 0.0223 and $0.0266 \mathrm{~nm}^{-1}$, the intensity peaks at $135^{\circ}$ and $-45^{\circ}$ (Figure 4.15B), which indicates that the particles align perpendicular to the field direction.

The goethite particles were embedded in a pNIPAM matrix. These macrogels were synthesized as described in Section 4.3.3.2 similar to Nack et al. [73]. Within this macrogel-even at a high field strength of about $550 \mathrm{mT}$-there is no visible alignment at room temperature (Figure 4.17A). The averaged normalized intensity at a defined wave vector range between 0.0223 and $0.0266 \mathrm{~nm}^{-1}$ results in a parallel line. Increasing the temperature to $30^{\circ} \mathrm{C}$, close to the LCST, makes an alignment of the particles possible (Figure 4.16B). An anisotropic pattern is directly visible in the SAXS data which leads to a peak at $-45^{\circ}$ and $135^{\circ} \mathrm{C}$ (Figure 4.15B). A further increase of the temperature does not lead to further alignment (Figures 4.15B and 4.16C). Returning to room temperature while under influence of the field shows that the particles stay aligned even after turning off the
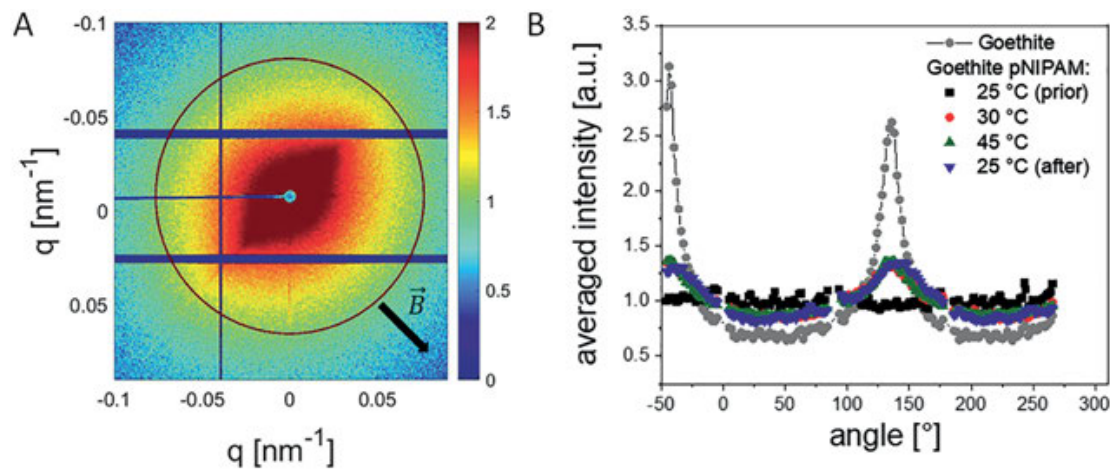

Figure 4.15: (A) U-SAXS of goethite particles in water in a field of about $550 \mathrm{mT}$. The field direction is indicated with a black arrow. The ring is a guideline for the eye. (B) Averaged intensity at a defined wave vector range, i.e., between 0.0223 and $0.0266 \mathrm{~nm}^{-1}$ versus angle for goethite particles in water and goethite particles in a pNIPAM matrix at $25^{\circ} \mathrm{C}$ prior to the first heating cycle, at $30^{\circ} \mathrm{C}, 45^{\circ} \mathrm{C}$ and at $25^{\circ} \mathrm{C}$ after the heating cycle. 


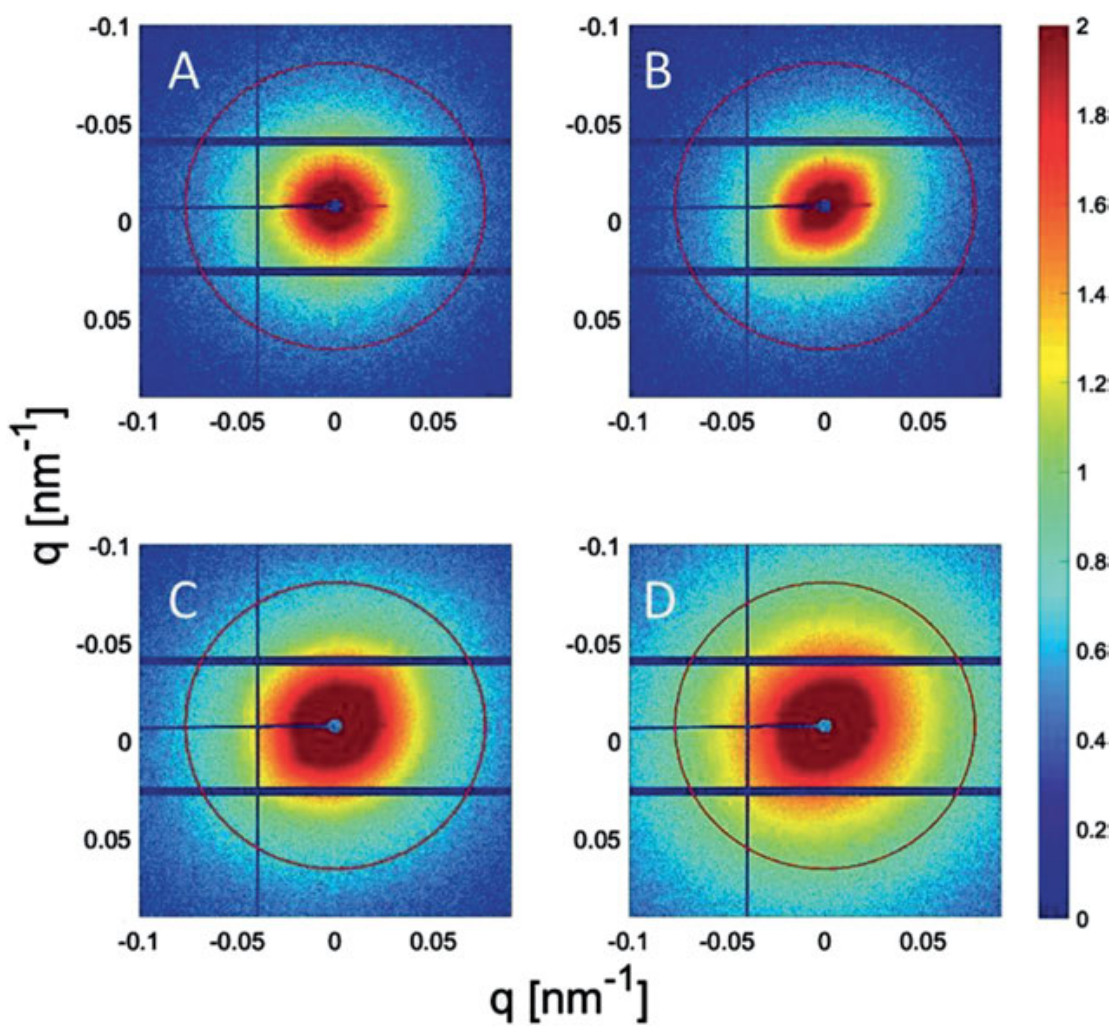

Figure 4.16: SAXS images of a pNIPAM macrogel with embedded goethite particles in a permanent magnetic field of about $550 \mathrm{mT}$ at (A) RT prior to the heating cycle, (B) $30^{\circ} \mathrm{C}$, (C) $45^{\circ} \mathrm{C}$ and (D) again at RT after the heating cycle.

field (Figures 4.15B and 4.16D). A detailed investigation on how this alignment affects the properties of the pNIPAM gel and the particle dynamics is running.

\subsubsection{Outlook: particles within network cavities}

When particles are embedded into a gel as described in Section 4.3.4, they are fixated in the matrix, i.e., the particles cannot move isolated from the matrix. The described particles can basically be split into three compartments: the magnetic nanoparticle, the silica shell and the hydrogel shell. The magnetic nanoparticle itself and the hydrogel have a distinct function in the composite material while the silica shell is used to create a stable composite material which becomes obsolete in the finished material. One approach to tune the composite can be the removal the silica shell [90-93], which should increase mobility of the magnetic particle and therefore open the opportunity to utilize applications that require mobile particles, e.g., for hyperthermia. 

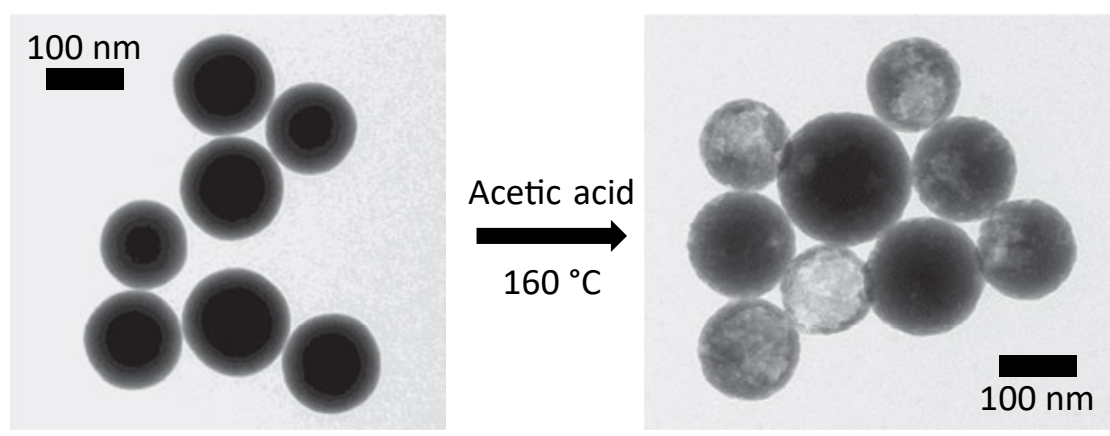

Figure 4.17: TE micrograph of the evolution of hollow silica spheres through etching with acetic acid before and after.

Silica shells are formed by a condensation reaction of SiOH. The most common reagent TEOS carries four such alcohol groups. Apart from condensation reactions these groups can also associate by hydrogen bond formation, which is less stable but significantly faster and the resulting bond-length is shorter. During the rapid formation of the silica particles the condensation typically is partial in the center. The degree of condensation typically increases closer to the surface of the particles because the increased curvature forces the $\mathrm{Si}$ to form the longer condensed $\mathrm{Si}-\mathrm{O}-\mathrm{Si}$-bonds. We attempted several approaches to dissolve/etch the silica shell [90-93]. The most commonly reported approach is using sodium hydroxide as etching agent which has the inherent disadvantage that it also catalyzes the condensation reaction [91]. This renders the whole process very difficult to control. Another seemingly more controlled process was the use of acetic acid [90]. The described approach is very straightforward: Bring a solution of silica or core-shell particles to $\mathrm{pH} 3.9$ and heat it to $160^{\circ} \mathrm{C}$ for $24 \mathrm{~h}$ in an autoclave. The first results can be seen in Figure 4.17 and show hollow silica spheres (B) in comparison to the particles before treatment (A). This approach lacks reproducibility and the results are very inconsistent leading us to abandoning this approach.

A combination of a very fine-tuned sodium hydroxide etching with a subsequent dialysis step yielded promising results at etching away about 95\% of the silica shell. This approach does not produce hollow spheres but removes the silica shell in its entirety. This process has the disadvantage that it has a direct influence on the swelling behavior of the gel material and-while being very reproducible-is therefore problematic to transfer into a practical application. A newer approach was recently discovered using ammonium fluoride to etch the silica shell [92]. Ammonium fluoride works as efficient as hydrofluoric acid while being far less dangerous to handle. First experiments showed a very clean etching of the silica shell while leaving the gel's swelling behavior unaffected. This approach will be further pursued in the project to create mobile particles in the center of a gel bead. 


\subsection{Hyperthermia-the step toward application}

Hyperthermia is a medical symptom describing an elevation of the temperature in the human body as a reaction to certain pathogens. Medical hyperthermia utilizes an external heating source to induce that overheating. The main disadvantage of the 'classic' approach to medical hyperthermia is that it requires methods that are either highly invasive in case of microwaves or need direct contact to the body in case of heating elements. Magnetic hyperthermia is an approach to realize specific and targeted heating while being minimally invasive and noncontact. Magnetic radiation of reasonable field strength is harmless to the human body and easily penetrates tissue without causing damage. To induce heat with magnetic radiation magnetic material must be brought to a target area. In our project we were not concerned with targeting an area but rather with the proof of concept of noncontact heating utilizing magnetic nanoparticles in an external ac field. For this purpose, we build a custom setup to measure heat induction in nanoparticle solutions. The core of the setup is a series resonant circuit which includes a $2 \Omega$ power resistor and is powered by an audio amplifier. The temperature is measured with a fiber optic thermometer directly in solution [66].

In general, there are two approaches how to evaluate acquired data from hyperthermia. The main approach to assess heating efficiency of magnetic material is a calorimetric approach that mainly takes the medium around the material into account while normalizing the data to the mass $m$ of particles used, the heat capacity of the medium $c$ and the slope of the temperature vs. time curve $\Delta T / \Delta t$ :

$$
\mathrm{SLP}=\frac{c}{m} \frac{\Delta T}{\Delta t}
$$

The intrinsic approach takes the actual magnetic characteristics of the material and the excitation field into account. The relation of specific loss power (SLP) and intrinsic loss power (ILP) can be described using the frequency $f$ and the strength $H$ of the excitation field:

$$
\mathrm{ILP}=\frac{\mathrm{SLP}}{f * H^{2}}
$$

The SLP can be directly accessed via temperature versus time measurements of a nanoparticle solution using the specific heat capacity of the solvent and/or matrix material. For comparability the data is usually normalized to the mass of particles used for a measurement.

Figure 4.18 shows heating versus time curves in dependence on the external magnetic field strength. The linear region in the first approximate $180 \mathrm{~s}$ can be utilized to calculate the SLP, which is shown in reliance to the respective field strength. The particles show a high heating power even at low frequent fields. This can be further tuned by optimizing the excitation frequency or increasing the viscosity of the solution. 


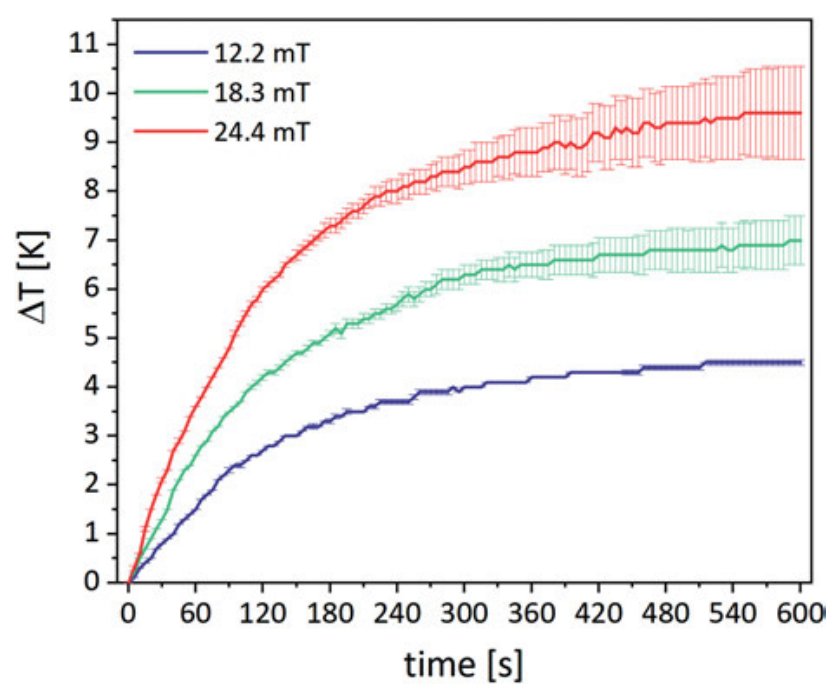

Figure 4.18: Temperature versus time curves comparing the heating ability of cobalt ferrite in pure water at different field strengths. With higher field strength the heating efficiency increases but also the error from coil heating.

pNIPAM can be synthesized as beads in aqueous solution and mixed with water dispersible particles. Figure 4.19 shows the hydrodynamic radius in dependence on the temperature for pure pNIPAM beads in water. A steep phase transition can be seen at roughly $30{ }^{\circ} \mathrm{C}$. As a proof of principle these beads were mixed with cobalt ferrite particles and measured in our setup.

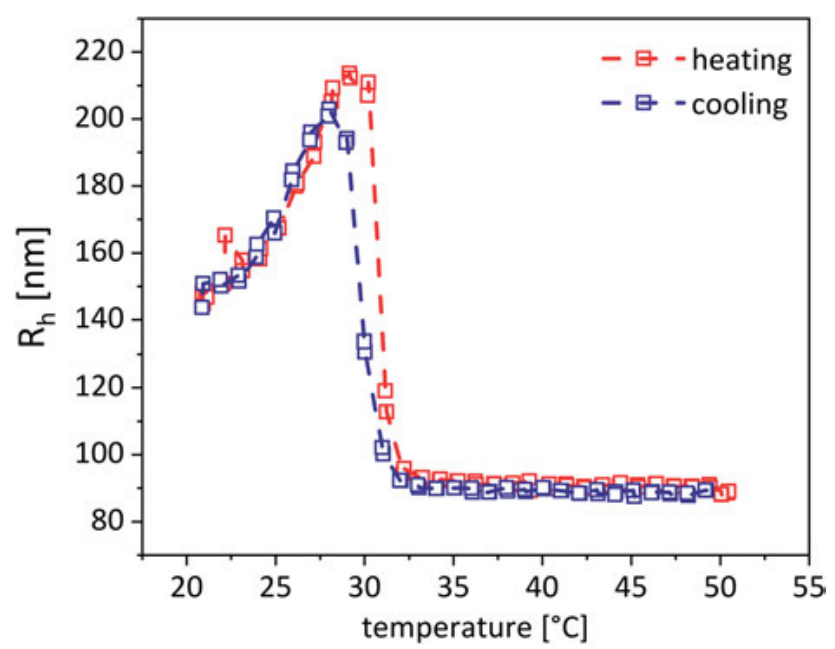

Figure 4.19: Temperature-dependent hydrodynamic radii of pNIPAM gel beads in water. A steep decrease of the hydrodynamic radius at around $30^{\circ} \mathrm{C}$ can be seen indicating the phase transition. 
Figure 4.20 exemplarily shows the heating versus time curves of two composite mixtures in comparison to a pure particle sample starting from ambient temperature. Evidently the composite mixture reaches roughly the double temperature increase while having about $60 \%$ of the particle content. This efficiency increase of about $300 \%$ can be explained by the increased Brownian relaxation time of the particles due to the higher viscosity of the solution. This increased relaxation time shifts the ideal excitation frequency of the particles to lower frequencies and therefore we get increased efficiency at the given frequency.

\subsection{Methods}

\subsubsection{TEM}

A drop of diluted particle suspension was deposited on a carbon coated copper grid. The solvent was removed by a filter paper. TE micrographs were recorded on a FEI Tecnai G2 spirit TWIN at an accelerating voltage of $120 \mathrm{kV}$.

\subsubsection{VSM}

The static magnetic characterization was conducted directly in solution using the commercially available EZ9 VSM system by Microsense in a range of $\pm 2.5 \mathrm{~T}$.

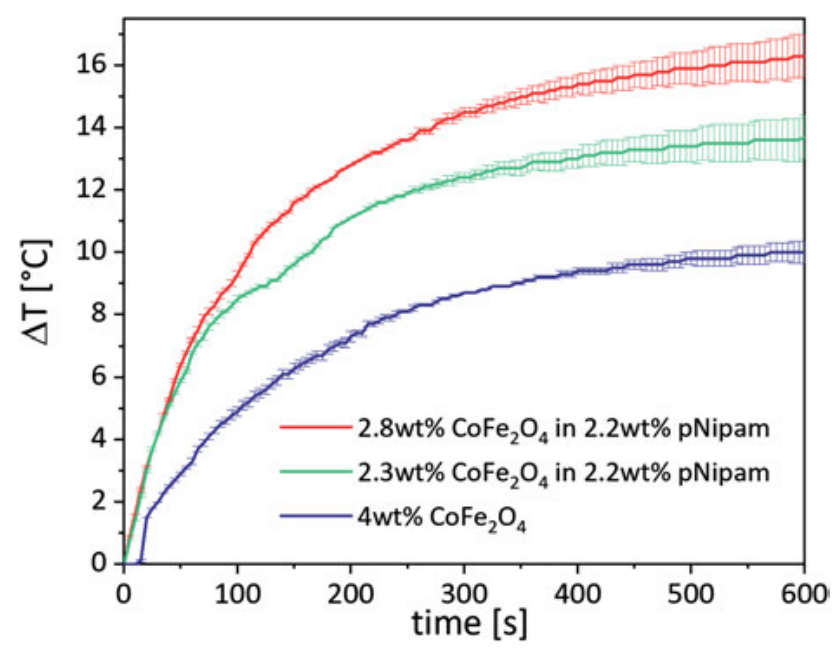

Figure 4.20: Temperature versus time curves comparing the heating ability of cobalt ferrite in pure water with cobalt ferrite mixed in a solution of pNIPAM gel beads with error bars. 


\subsubsection{XRD}

The sample was washed to neutral $\mathrm{pH}$. Then about $100 \mu \mathrm{L}$ of the particle dispersion were deposited on a silicon waver and dried in air. The X-ray diffraction was done on a Philips X'Pert PRO MPD. The radiation was $\mathrm{Cu}-\mathrm{K}_{\alpha}$ with a wavelength of $1.54 \AA$ A. For the evaluation of the crystal phases present, we used the software Highscore X'pert PRO by PanAnalytical with the PDF Nr. 00-034-1266 for akaganeite, PDF Nr. 00-022-1086 for cobalt ferrite, PDF Nr. 00-024-0072 for hematite, PDF Nr. 01-081-0462 for goethite and PDF Nr. 01-086-1344 for magnetite. All samples were background corrected to compensate for X-ray fluorescence at the given X-ray wavelength.

\subsubsection{DLS}

The dynamic light scattering (DLS) measurements were performed with an ALV/CGS-3 Compact Goniometer-System using an ALV/LSE-5004 Multiple Tau Digital Correlator (V.1.7.9.) in combination with a COBOLT SAMBA 50 laser (Nd:YAG, $532 \mathrm{~nm}, 400 \mathrm{~mW}$ ) and the ALV Digital Correlator Software 3.0. The measuring angle was set to $90^{\circ}$ for all measurements and every individual measurement was conducted for $60 \mathrm{~s}$. The sample vials consisted of quartz glass and were placed into a measurement cell filled with toluene. The temperature-dependent viscosity and refractive index of the solvents were automatically corrected according to tabulated values. The toluene bath and thus the samples were tempered by a JULABO F25 thermostat working with a mixture of water and ethylene glycol and delivering a temperature accuracy of $0.01^{\circ} \mathrm{C}$.

\subsubsection{Hyperthermia}

Hyperthermia was measured with a custom-built coil setup. The frequency was set using the STEMlab web interface of the red pitaya amplified by a t.amp TSA 2200 audio amplifier from Thomann powering a series resonant circuit including an additional $2 \Omega$ power resistor. The factual field strength was calculated directly from the measured current driving the excitation coil. The current was measured with a Sensitec 3025 sensor. The signal was processed within STEMlab of the red pitaya. The temperature was measured with a fiberoptic gallium arsenide measurement device from Optocon. The sample holder is a 3D-printed ABS piece to fit $5 \mathrm{~mL}$ rotilab glass vials from Carl Roth and is thermally insulated by a $2 \mathrm{~mm}$ water-cooled polystyrene hose.

\subsubsection{U-SAXS}

The U-SAXS measurements were performed at the Coherence Beamline P10 at the German Synchrotron Facility (DESY) in Hamburg. The energy was set to $7.049 \mathrm{keV}$, 
which lies under the absorption edge of iron. The distance from detector to sample was about $21.2 \mathrm{~m}$. An Eiger 4M detector with $2070 \times 2167$ pixels was used with a pixel size of about $75 \times 75 \mu \mathrm{m}^{2}$. The samples were filled in Quartz capillaries prior to the measurement and sealed with hot glue. For the low magnetic field strengths, a coil setup was utilized generating a DC magnetic field up to $100 \mathrm{mT}$. For stronger magnetic field strengths permanent magnets were used. The field strength was measured by a hall effect magnetometer.

\subsection{Summary and outlook}

We succeeded in the synthesis of a variety of iron oxide phases in different sizes and shapes with unique magnetic properties. The iron oxides are usually very stable in their respective oxidation phases and maintain their respective properties for months. All particles can be additionally coated with a silica shell which further prevents agglomeration and offers the possibility for further functionalization via the very flexible silicon hydroxide function on the outer shell. We additionally showed the combination of our particle systems with pNIPAM as a proof-of-principle of a composite material for how the thermoresponsive matrix can be influenced by a magnetic field. A variety of different analysis methods could be applied to the particles.

Magnetite generally has very good magnetic properties but proved to be very sensitive to handle. We could show with various scattering techniques that impurities of maghemite or magnetically far inferior hematite were a very common byproduct of the synthesis. We succeeded to establish a hydrothermal route that enables us to synthesize phase pure magnetite nanoparticles not only from pre-prepared akageneite precursors but also from impure samples as a means for purification. These akaganeite precursors are very simple and controlled in their synthetic route and can also be used to create antiferromagnetic goethite particles which have unique magnetic properties as well. Cobalt ferrite as a hard-magnetic counterpart to magnetite can also be synthesized straightforward and was stabilized for months in watery dispersion.

All mentioned particles can be reliably coated with a silica shell that is tunable in its size. This shell offers the possibility of a wide range of functionalization through silane chemistry. The silica shell itself seems to offer quite sophisticated properties regarding biocompatibility and opsonization behavior in biological fluids like serum or blood. We could show that our cobalt ferrite nanoparticles express a seemingly low toxicity under $48 \mathrm{~h}$ which could be further reduced through the silica shell. Preliminary tests showed that proteins in fetal calf serum coordinate well around silica coated cobalt ferrite particles which has potential in a future application to mask particles from an answer of the bodily immune system.

An array of interactions between our different particle systems and the thermoresponsive matrix pNIPAM could be observed and reported in this work. pNIPAM has a significant influence on the orientational behavior which can be switched by a 
temperature change. We could also induce enough heat to achieve the phase transition via an alternating current magnetic field into a gel without direct contact.

The proofs of principle presented in this work will be further pursued in the future. The cobalt ferrite particles will be tuned in their stability in biological fluids as serum and blood. Hemocompatibility tests as well as toxicity assays with different cell lines are planned as the next step. Different sizes of cobalt ferrite particles and a comparative toxicological assay dependent on the particle size and magnetic properties. Parallel to biocompatibility in general, cobalt ferrite and magnetite nanoparticles will be further examined on their hyperthermia behavior in gel matrices with a special focus on the difference between particles that are just mixed into a gel and particles that are covalently bound into the network. Especially temperature-dependent dynamic magnetic analysis is expected to deliver very interesting insights to particle matrix interactions. Further X-ray scattering experiments on goethite nanorods are planned to get an even better insight into the orientational behavior and the change of that orientational behavior inside different gel matrices.

Acknowledgment: The authors would also like to thank various work groups inside the SPP1681 for the fruitful cooperation that lead to different publications during the time of the project. The authors would like to acknowledge Andreas Weidner and the group of Sylvio Dutz from Ilmenau for the cooperation in the synthesis of multicore particles. The authors would like to thank Sebastian Draack and the groups of Thilo Viereck and Frank Ludwig for fruitful discussions and mutual publication of the research. The authors would also like to thank Ralf P. Friedrich and the group of Christoph Alexiou for much insight into biocompatibility of nanoparticles and work toward a publication in cooperation with Sebastian Draack. The authors would like to thank Almut Barck and Margarethe Fritz for measuring XRD. The authors acknowledge Fabian Westermeier and Michael Sprung for their help during beamtime at DESY P10.

Author contributions: All the authors have accepted responsibility for the entire content of this submitted manuscript and approved submission.

Research funding: The Deutsche Forschungsgemeinschaft is kindly acknowledged for funding our project under the SPP 1681 "Field Controlled Particle-Matrix Interactions" with the Project FI 1235/2-1 and FI 1235/2-2. Stephan Hinrichs would like to acknowledge the doctoral scholarship of the University of Hamburg for funding the first funding period of his work.

Conflict of interest statement: The authors declare no conflicts of interest regarding this article.

\section{References}

1. Lattermann G, Krekhova M. Thermorevers Ferrogels Macromol Rapid Commun 2006;27:1373-9.

2. Messing R, Schmidt AM. Perspectives for the mechanical manipulation of hybrid hydrogels. Polym Chem 2011;2. https://doi.org/10.1039/c0py00129e. 
3. Thevenot J, Oliveira H, Sandre O, Lecommandoux S. Magnetic responsive polymer composite materials. Chem Soc Rev 2013;42:7099-116.

4. Menzel AM. Tuned, driven, and active soft matter. Phys Rep 2015;554:1-45.

5. Gil ES, Hudson SM. Stimuli-responsive polymers and their bioconjugates. Prog Polym Sci 2004;29: 1173-222.

6. Smith AE, Xu X, McCormick CL. Stimuli-responsive amphiphilic (co)polymers via RAFT polymerization. Prog Polym Sci 2010;35:45-93.

7. de Las Heras Alarcon C, Pennadam S, Alexander C. Stimuli responsive polymers for biomedical applications. Chem Soc Rev 2005;34:276-85.

8. Calvert P. Hydrogels for soft machines. Adv Mater 2009;21:743-56.

9. Li Y, Huang G, Zhang X, Li B, Chen Y, Lu T, et al. Magnetic hydrogels and their potential biomedical applications. Adv Funct Mater 2013;23:660-72.

10. Jordan A, Scholz R, Maier-Hauff K, Johannsen J, Wust P, Nadobny J, et al. Presentation of a new magnetic field therapy system for the treatment of human solid tumors with magnetic fluid hyperthermia. J Magn Magn Mater 2001;225. https://doi.org/10.1016/s0304-8853(00)01239-7.

11. Yadavalli T, Ramasamy S, Chandrasekaran G, Michael I, Therese HA, Chennakesavulu R. Dual responsive PNIPAM-chitosan targeted magnetic nanopolymers for targeted drug delivery. J Magn Magn Mater 2015;380:315-20.

12. Büscher K, Helm CA, Gross C, Glöckl G, Romanus E, Weitschies W. Nanoparticle composition of a ferrofluid and its effects on the magnetic properties. Langmuir 2004;20. https://doi.org/10.1021/ la030261x.

13. Hergt R, Dutz $S$, Roder M. Effects of size distribution on hysteresis losses of magnetic nanoparticles for hyperthermia. J Phys Condens Matter 2008;20:385214.

14. Rosensweig RE. Heating magnetic fluid with alternating magnetic field. J Magn Magn Mater 2002; 252:370-4.

15. Ozaki M, Egami T, Sugiyama N, Matijević E. Agglomeration in colloidal hematite dispersions due to weak magnetic interactions: II. The effects of particle size and shape. J Colloid Interface Sci 1988; 126:212-9.

16. Ozaki M, Suzuki H, Takahashi K, Matijević E. Reversible ordered agglomeration of hematite particles due to weak magnetic interactions. J Colloid Interface Sci 1986;113:76-80.

17. Lee N, Kim H, Choi SH, Park M, Kim D, Kim H-C, et al. Magnetosome-like ferrimagnetic iron oxide nanocubes for highly sensitive MRI of single cells and transplanted pancreatic islets. Proc Natl Acad Sci USA 2011;108:2662-7.

18. Materia ME, Guardia P, Sathya A, Pernia Leal M, Marotta R, Di Corato R, et al. Mesoscale assemblies of iron oxide nanocubes as heat mediators and image contrast agents. Langmuir 2015;31:808-16.

19. Wang C, Peng S, Lacroix L-M, Sun S, et al. Synthesis of high magnetic moment CoFe nanoparticles via interfacial diffusion in core/shell structured Co/Fe nanoparticles. Nano Res 2010;2:380-5.

20. Santoyo Salazar J, Perez L, de Abril O, Truong Phuoc L, Ihiawakrim D, Vazquez M, et al. Magnetic iron oxide nanoparticles in $10-40 \mathrm{~nm}$ range: composition in terms of magnetite/maghemite ratio and effect on the magnetic properties. Chem Mater 2011;23:1379-86.

21. Hufschmid R, Arami H, Ferguson RM, Gonzales M, Teeman E, Brush LN, et al. Synthesis of phasepure and monodisperse iron oxide nanoparticles by thermal decomposition. Nanoscale 2015;7: 11142-54.

22. Palanisamy S, Wang YM. Superparamagnetic iron oxide nanoparticulate system: synthesis, targeting, drug delivery and therapy in cancer. Dalton Trans 2019;48:9490-515.

23. Massart R, Dubois E, Cabuil V, Hasmonay E. Preparation and properties of monodisperse magnetic fluids. J Magn Magn Mater 1995;149:1-5.

24. Kaman O, Kulickova J, Herynek V, Koktan J, Maryško M, Dědourková T, et al. Preparation of Mn-Zn ferrite nanoparticles and their silica-coated clusters: magnetic properties and transverse relaxivity. J Magn Magn Mater 2017;427:251-7. 
25. Hayashi H, Hakuta Y. Hydrothermal synthesis of metal oxide nanoparticles in supercritical water. Materials 2010;3:3794-817.

26. Cornell RM, Schwertmann U. Adsorption of ions and molecules. In: The iron oxides. Weinheim: Wiley-VCH Verlag GmbH \& Co. KGaA; 2003:253-96 pp.

27. Thies-Weesie DME, de Hoog JP, Mendiola MHH, Petukhov AV, Vroege GJ. Synthesis of goethite as a model colloid for mineral liquid crystals. Chem Mater 2007;19:5538-46.

28. Rădiţoiu V, Diamandescu L, Cosmin Corobea M, Rădiţoiu A, Popescu-Pogrion N, Nicolae CA. A facile hydrothermal route for the synthesis of $\alpha-\mathrm{FeOOH}$ with controlled morphology. J Cryst Growth 2012; 348:40-6.

29. Zamiri R, Ahangar HA, Zakaria A, Zamiri G, Bahari HR, Drummen GPC. Hydrothermal synthesis of goethite $(\alpha-\mathrm{FeOOH})$ nanorods in the presence of ethylenediamine:thiourea. J Nano Res 2014;16: 1-10.

30. Frens $\mathrm{G}$. Controlled nucleation for the regulation of the particle size in monodisperse gold suspensions. Nat Phys Sci (Lond) 1973;241:20-2.

31. Leff DV, Ohara PC, Heath JR, Gelbart WM. Thermodynamic control of gold nanocrystal size: experiment and theory. J Phys Chem 1995;99:7036-41.

32. Hinrichs S, Hankiewicz B. Goethite nanorods: synthesis and investigation of the size effect on their orientation within a magnetic field by SAXS. Nanomaterials 2020;10:2526.

33. Sugimoto $\mathrm{S}$, Wang $\mathrm{Y}$, Itoh $\mathrm{H}$, Muramatsu $A$. Systematic control of size, shape and internal structure of monodisperse $\alpha-\mathrm{Fe}_{2} \mathrm{O}_{3}$ particles. Colloids Surf, A Physicochem Eng Asp 1998;265-79.

34. Malik V, Grobety B, Trappe V, Dietsch H, Schurtenberger P. A closer look at the synthesis and formation mechanism of hematite nanocubes. Colloids Surf A-Physicochem Eng Asp 2014;445: 21-9.

35. Ozaki M, Kratohvil S, Matijevic E. Formation of monodispersed spindle-type hematite particles. J Colloid Interface Sci 1984;102:146-51.

36. Märkert C, Fischer B, Wagner J. Small-angle scattering from spindle-shaped colloidal hematite particles in external magnetic fields. J Appl Crystallogr 2011;44:441-7.

37. Pu ZF, Cao MH, Jing Y, Hu C. Controlled synthesis and growth mechanism of hematite nanorhombohedra, nanorods and nanocubes. Nanotechnology 2006;17:799-804.

38. Harraz FA. Polyethylene glycol-assisted hydrothermal growth of magnetite nanowires: synthesis and magnetic properties. Phys E Low-dimens Syst Nanostruct 2008;40:3131-6.

39. Sugimoto T, Muramatsu A. Formation mechanism of monodispersed $\alpha-\mathrm{Fe}_{2} \mathrm{O}_{3}$ particles in dilute $\mathrm{FeCl}_{3}$ solutions. J Colloid Interface Sci 1996;184:626-38.

40. Wiogo H, Lim M, Munroe P, Amal R. Understanding the formation of iron oxide nanoparticles with acicular structure from iron(III) chloride and hydrazine monohydrate. Cryst Growth Des 2011;11: 1689-96.

41. Yang W-H, Lee C-F, Tang HY, Shieh D-B, Yeh C-S. Iron oxide nanopropellers prepared by a lowtemperature solution approach. J Phys Chem B 2006;110:14087-91.

42. Mertelj A, Lisjak D, Drofenik M, Čopič M. Ferromagnetism in suspensions of magnetic platelets in liquid crystal. Nature 2013;504:237-41.

43. Lemaire BJ, Davidson P, Panine P, Jolivet JP. Magnetic-field-induced nematic-columnar phase transition in aqueous suspensions of goethite $(\alpha-\mathrm{FeOOH})$ nanorods. Phys Rev Lett 2004;93: 267801.

44. Sánchez-Ferrer A, Mezzenga R, Dietsch H. Orientational behavior of ellipsoidal silica-coated hematite nanoparticles integrated within an elastomeric matrix and its mechanical reinforcement. Macromol Chem Phys 2011;212:627-34.

45. Haberl JM, Sanchez-Ferrer A, Mihut AM, Dietsch H, Hirt AM, Mezzenga R. Liquid-crystalline elastomer-nanoparticle hybrids with reversible switch of magnetic memory. Adv Mater 2013;25: 1787-91. 
46. Ozaki M, Matijević E. Preparation and magnetic properties of monodispersed spindle-type $\gamma-\mathrm{Fe}_{2} \mathrm{O}_{3}$ particles. J Colloid Interface Sci 1985;107:199-203.

47. Ngo AT, Pileni MP. Cigar-shaped ferrite nanocrystals: orientation of the easy magnetic axes. J Appl Phys 2002;92:8-4649.

48. Bee A, Massart R, Neveu S. Synthesis of very fine maghemite particles. J Magn Magn Mater 1995; 149:6-9.

49. Graf C, Vossen DLJ, Imhof A, van Blaaderen A. A general method to coat colloidal particles with silica. Langmuir 2003;19:6693-700.

50. Zou J, Peng YG, Tang YY. A facile bi-phase synthesis of $\mathrm{Fe}_{3} \mathrm{O}_{4} @ \mathrm{SiO}_{2}$ core-shell nanoparticles with tunable film thicknesses. RSC Adv 2014;4:9693-700.

51. Zhang L, Chen L, Qan Q-H. Preparation of uniform magnetic microspheres through hydrothermal reduction of iron hydroxide nanoparticles embedded in a polymeric matrixes. Chem Mater 2008; 20:3345-53.

52. Crassous JJ, Mihut AM, Dietsch H, Pravaz O, Ackermann-Hirschi L, Hirt AM, et al. Advanced multiresponsive comploids: from design to possible applications. Nanoscale 2014;6:8726-35.

53. Huang KC, Ehrman SH. Synthesis of iron nanoparticles via chemical reduction with palladium ion seeds. Langmuir 2007;23:1419-26.

54. Rozenson I, Heller-Kallai L. Reduction and oxidation of $\mathrm{Fe}^{3+}$ in dioctahedral smectites-1: reduction with hydrazine and dithionite. Clay Clay Miner 1976;24:271-82.

55. Anik T, Touhami ME, Himm K, Schireen S, Belkhmima RA, Abouchane M, et al. Influence of pH solution on electroless copper plating using sodium hypophosphite as reducing agent. Int J Electrochem Sci 2012;7:2009-18.

56. Klačanová K, Fodran P, Šimon P, Rapta P, Boča R, Jorik V, et al. Formation of Fe(0)-nanoparticles via reduction of $\mathrm{Fe}(\mathrm{II})$ compounds by amino acids and their subsequent oxidation to iron oxides. J Chem 2013;2013:1-10.

57. lida H, Nakanishi T, Takada H, Osaka T. Preparation of magnetic iron-oxide nanoparticles by successive reduction-oxidation in reverse micelles: effects of reducing agent and atmosphere. Electrochim Acta 2006;52:292-6.

58. Hayes PC, Algie SH. Process principles in minerals and materials production. Cincinnati: Hayes Publishing; 1993: $660 \mathrm{ff} p$.

59. Hinrichs S, Nun N, Fischer B. Synthesis and characterization of anisotropic magnetic hydrogels. J Magn Magn Mater 2017;431:237-40.

60. Peng Z, Wu M, Xiong Y, Wang J, Chen Q. Synthesis of magnetite nanorods through reduction of $\beta$-FeOOH. Chem Lett 2005;34:636-7.

61. Fan R, Chen XH, Gui Z, Liu L, Chen ZY. A new simple hydrothermal preparation of nanocrystalline magnetite $\mathrm{Fe}_{3} \mathrm{O}_{4}$. Mater Res Bull 2001;36:497-502.

62. Nemati Z, Salili SM, Alonso J, Ataie A, Das R, Phan MH, et al. Superparamagnetic iron oxide nanodiscs for hyperthermia therapy: does size matter? J Alloys Compd 2017;714:709-14.

63. Dutz S, Clement JH, Eberbeck D, Gelbrich T, Hergt R, Müller R, et al. Ferrofluids of magnetic multicore nanoparticles for biomedical applications. J Magn Magn Mater 2009;321:1501-4.

64. Grafe C, von der Luhe M, Weidner A, Globig P, Clement JH, Dutz S, et al. Protein corona formation and its constitutional changes on magnetic nanoparticles in serum featuring a

polydehydroalanine coating: effects of charge and incubation conditions. Nanotechnology 2019; 30:1-15.

65. Nappini S, Magnano E, Bondino F, Píš I., Barla A., Fantechi E, et al. Surface charge and coating of $\mathrm{CoFe}_{2} \mathrm{O}_{4}$ nanoparticles: evidence of preserved magnetic and electronic properties. J Phys Chem C 2015;119:25529-41.

66. Lucht N, Friedrich RP, Draack S, Alexiou C, Viereck T, Ludwig F, et al. Biophysical characterization of (Silica-coated) cobalt ferrite nanoparticles for hyperthermia treatment. Nanomaterials 2019;9: $1-13$. 
67. Draack S, Lucht N, Remmer H, Martens M., Fischer B., Schilling M, et al. Multiparametric magnetic particle spectroscopy of $\mathrm{CoFe}_{2} \mathrm{O}_{4}$ nanoparticles in viscous media. J Phys Chem C 2019;123: 6787-801.

68. Remmer H, Dieckhoff J, Tschöpe A, Roeben E, Schmidt AM, Ludwig F. Dynamics of $\mathrm{CoFe}_{2} \mathrm{O}_{4}$ singlecore nanoparticles in viscoelastic media. Physics Procedia 2015;75:1150-7.

69. Müller R, Zhou M, Liebert T, Landers J, Salamon S, Webers S, et al. Mobility investigations of magnetic nanoparticles in biocomposites. Mater Chem Phys 2017;193:364-70.

70. Bossi E, Zanella D, Gornati R, Bernardini G. Cobalt oxide nanoparticles can enter inside the cells by crossing plasma membranes. Sci Rep 2016;6:1-9.

71. Satarkar NS, Biswal D, Hilt IZ. Hydrogel nanocomposites: a review of applications as remote controlled biomaterials. Soft Matter 2010;6:2364-71.

72. Witt MU, Hinrichs S, Moller N, Backes S, Fischer B, von Klitzing R. Distribution of $\mathrm{CoFe}_{2} \mathrm{O}_{4}$ nanoparticles inside PNIPAM-based microgels of different cross-linker distributions. J Phys Chem B 2019;123: 2405-13.

73. Nack A, Seifert J, Passow C, Wagner J. Hindered nematic alignment of hematite spindles in poly(N-isopropylacrylamide) hydrogels: a small-angle X-ray scattering and rheology study. J Appl Crystallogr 2018;51:87-96.

74. Messing R, Frickel N, Belkoura L, Strey R, Rahn H, Odenbach S. Cobalt ferrite nanoparticles as multifunctional cross-linkers in PAAm ferrohydrogels. Macromolecules 2011;44:2990-9.

75. Hu Z, Huang G. A new route to crystalline hydrogels, guided by a phase diagram. Angew Chem Int Ed Engl 2003;42:4799-802.

76. Roeder L, Reckenthäler M, Belkoura L, Roitsch S, Strey R, Schmidt AM. Covalent ferrohydrogels based on elongated particulate cross-linkers. Macromolecules 2014;47:7200-7.

77. Acciaro R, Gilanyi T, Varga I. Preparation of monodisperse poly(N-isopropylacrylamide) microgel particles with homogenous cross-link density distribution. Langmuir 2011;27:7917-25.

78. Lucht N, Eggers S, Abetz V. Cononsolvency in the 'drunken' state: the thermoresponsiveness of a new acrylamide copolymer in water-alcohol mixtures. Polym Chem 2017;8:1196-205.

79. Bozorg M, Hankiewicz B, Abetz V. Soft Matter 2020;16:1066-81.

80. Karg M, Pastoriza-Santos I, Liz-Marzan LM, Hellweg T. A versatile approach for the preparation of thermosensitive PNIPAM core-shell microgels with nanoparticle cores. Chem Phys Chem 2006;7: 2298-301.

81. Karg M, Wellert S, Prevost S, Schweins R, Dewhurst C, Liz-Marzán LM, et al. Well defined hybrid PNIPAM core-shell microgels: size variation of the silica nanoparticle core. Colloid Polym Sci 2011; 289:699-709.

82. Nun N, Hinrichs S, Schroer MA, Sheyfer D, Grübel G, Fischer B. Tuning the size of thermoresponsive poly(N-isopropyl acrylamide) grafted silica microgels. Gels 2017;3:3-13.

83. Hu Z, Huang G. A new route to crystalline hydrogels, guided by a phase diagram. Angew Chem Int Ed Engl 2003;42:4799-802.

84. Migneault I, Dartiguenave C, Bertrand MJ, Waldron KC. Glutaraldehyde: behavior in aqueous solution, reaction with proteins, and application to enzyme crosslinking. Biotechniques 2004;37: 798-802.

85. Wu X, Pelton RH, Hamielec AE, Woods DR, McPhee W. The kinetics of poly(N-isopropylacrylamide) microgel latex formation. Colloid Polym Sci 1994;272:467-77.

86. Ozaki M. Preparation and properties of well-defined magnetic particles. MRS Bull 1989;14:35-40.

87. Wagner J, Markert C, Fischer B, Müller L. Direction dependent diffusion of aligned magnetic rods by means of $x$-ray photon correlation spectroscopy. Phys Rev Lett 2013;110:048301-1-048301-5.

88. Landers J, Roeder L, Salamon S, Jamet JP, Panine P, Dozov I, et al. Particle-matrix interaction in cross-linked PAAm-hydrogels analyzed by Mössbauer spectroscopy. J Phys Chem C 2015;119: 20642-8. 
89. Lemaire BJ, Davidson P, Ferré J, Jamet JP, Panine P, Dozov I, et al. Outstanding magnetic properties of nematic suspensions of goethite (alpha-FeOOH) nanorods. Phys Rev Lett 2002;88:12-125507.

90. Ding H, Zhang Y, Xu S, Li G. A wrinkle to sub-100 nm yolk/shell $\mathrm{Fe}_{3} \mathrm{O}_{4} @ \mathrm{SiO}_{2}$ nanoparticles. Nano Research 2016;9:3632-43.

91. Meng Q, Xiang S, Zhang K, Wang M, Bu X., Xue P, et al. A facile two-step etching method to fabricate porous hollow silica particles. J Colloid Interface Sci 2012;384:22-8.

92. Kalem S. Synthesis of ammonium silicon fluoride cryptocrystals on silicon by dry etching. Appl Surf Sci 2004;236:336-41.

93. Liu Z, Chen L, Ye X, Yu H, Li J, Zeng F. Selective basic etching of bifunctional core-shell composite particles for the fabrication of organic functionalized hollow mesoporous silica nanospheres. New J Chem 2016;40:825-31. 